\title{
ALS-linked KIF5A $\Delta$ Exon27 mutant causes neuronal toxicity through gain of function
}

Authors: Devesh C. Pant ${ }^{1 \dagger}$, Janani Parameswaran ${ }^{1 \dagger}$, Lu Rao ${ }^{2}$, Liang Shi ${ }^{1}$, Ganesh Chilukuri ${ }^{1}$, Zachary T. McEachin ${ }^{1,3}$, Jonathan Glass ${ }^{4}$, Gary J. Bassell ${ }^{1}$, Arne Gennerich ${ }^{2}$, Jie Jiang ${ }^{1 *}$

\section{Affiliations:}

${ }^{1}$ Department of Cell Biology, Emory University, Atlanta GA 30322, USA. ${ }^{2}$ Department of Anatomy and Structural Biology and Gruss Lipper Biophotonics Center, Albert Einstein College of Medicine, Bronx, NY 10461, USA. ${ }^{3}$ Department of Human Genetics, Emory University, Atlanta, GA 30322, USA. ${ }^{4}$ Department of Neurology, Emory University School of Medicine, Atlanta, GA 30322, USA.

†These authors contributed equally to this work.

14 Correspondence should be addressed to Jie Jiang (jie.jiang@emory.edu)

Tel: +1404-727-1597; Fax: +1404-727-6256

\section{Running Title: Gain of toxicity in KIF5A-associated ALS}

\section{Abstract}

Mutations in the human kinesin family member 5A (KIF5A) gene were recently identified as a genetic cause of amyotrophic lateral sclerosis (ALS). Several KIF5A ALS variants cause exon 27 skipping and produce motor proteins with an altered C-terminal tail (referred to as $\Delta$ Exon27). However, the underlying pathogenic mechanism is still unknown. In this study, we performed a comprehensive analysis of $\Delta$ Exon 27 at the single-molecule, cellular, and organism levels. Our results show that $\Delta$ Exon27 is prone to form cytoplasmic aggregates and is neurotoxic. The mutation relieves motor autoinhibition and increases motor self-association, leading to drastically enhanced processivity on microtubules. Finally, ectopic expression of $\Delta$ Exon 27 in

Drosophila melanogaster causes wing defects, motor impairment, paralysis and premature death.

Our results suggest gain of function as an underlying disease mechanism in KIF5A-associated ALS.

Keywords: Aggregation, ALS, Autoinhibition, Gain of toxicity, KIF5A

\section{Introduction}

Amyotrophic Lateral Sclerosis (ALS) is a progressive neurodegenerative disease characterized by loss of upper and lower motor neurons (MNs) leading to paralysis and death within 3-5 years after diagnosis (Chia et al, 2018). Most of ALS patients are classified as sporadic ALS (sALS), 
while about $10 \%$ of patients show a clear family history (fALS). Although varying in disease onset and duration, fALS and sALS patients cannot be differentiated by their clinical features. The mechanisms of disease pathogenesis leading to the exclusive demise of MNs remain unclear and there is no effective therapy. Over 30 genes have been linked to ALS, and several of these genes such as PFN1 (profilin 1) and TUBA4A (tubulin, alpha 4A) are involved in cytoskeletal function and intracellular transport (Brenner \& Weishaupt, 2019). Motor neurons are highly polarized cells with neurites that can exceed 1 meter in length. Various materials such as mRNA, proteins, lipids, membrane-bound vesicles, and organelles commute between cell bodies and synaptic terminals as they are transported along microtubules by two major families of motor proteins. Kinesin family (KIF) proteins mediate anterograde transport away from the cell body to neurite terminals whereas cytoplasmic dynein-1 drives the retrograde transport in the opposite direction. In 2018, two studies reported that mutations in KIF5A (OMIM\# 602821) cause ALS in European and North American cohorts, further highlighting the role of intracellular transport disruption in disease pathogenesis (Brenner et al, 2018; Nicolas et al, 2018). Additional ALS patients with KIF5A mutations were subsequently identified in Asian cohorts (Faruq et al, 2019; Nakamura et al, 2021; Naruse et al, 2021; Zhang et al, 2019).

Human KIF5A, together with KIF5B and KIF5C, belongs to the kinesin-1 family and is one of the founding members of the kinesin superfamily with a total of 45 members (Hirokawa et al, 2009). The KIF5A motor complex is a heterotetramer consisting of two kinesin heavy chains (KHCs) and two kinesin light chains (KLCs). It uses the energy derived from ATP binding and hydrolysis to transport a variety of cargos processively (the ability to take hundreds of steps along microtubules before dissociation) to the plus-ends of microtubules. Studies in animal models support that KIF5A has essential functions in the development and functioning of the nervous system (Kanai et al, 2000; Tanaka et al, 1998; Xia et al, 2003). The KIF5A KHC contains an $\mathrm{N}$-terminal catalytic motor domain, an $\alpha$-helical stalk region, and a $\mathrm{C}$-terminal tail

66 region (Fig. 1A). KIF5A was previously identified as the causative gene for hereditary spastic 67 paraplegia-10 (SPG10) and Charcot-Marie-Tooth type 2 (CMT2), with mutations mostly

68 located in the N-terminal motor domain (Blair et al, 2006; Crimella et al, 2012; Fichera et al, 69 2004). Moreover, de novo heterozygous frameshift rare variants in the C-terminal domain of 70 KIF5A were reported to be associated with neonatal intractable myoclonus (NEIMY), a severe 
71 infantile-onset neurologic disorder characterized by myoclonic seizures, hypotonia, dysphagia,

72 and early development arrest (Duis et al, 2016; Rydzanicz et al, 2017). Interestingly, the ALS-

73 associated variants reported in KIF5A are mainly clustered at the $5^{\prime}$ and $3^{\prime}$ splice junctions of

74 exon 27 (Brenner et al., 2018; Nicolas et al., 2018). These variants cause a complete skipping of

75 exon 27, yielding a protein with the normal C-terminal 34 amino acids replaced with 39 aberrant

76 amino acids (referred to as $\Delta$ Exon27 hereafter) (Fig. 1A).

78 Currently, the molecular mechanism of $\triangle$ Exon27 underlying ALS pathogenesis is unclear. All known KIF5A ALS variants are autosomal dominant, and it is hypothesized that defective KIF5A alleles may lead to dysfunctional kinesin and impaired cargo transport. Alternatively, the altered $\mathrm{C}$-terminal tail in the $\Delta$ Exon 27 mutant might confer detrimental effects through a toxic gain-of-function mechanism. In this study, we performed a comprehensive analysis of $\Delta$ Exon 27 using genetic, biochemical, and single-molecule methods. We find that $\Delta$ Exon27 is prone to aggregation formation and is neurotoxic. Transgenic Drosophila expressing $\Delta$ Exon27 display wing deficits, motor impairment, paralysis, and premature death. Mechanistically, $\Delta$ Exon27 is constitutively active, displays increased motor self-association and drastically enhanced processivity on microtubules. Together, these results suggest the KIF5A $\Delta$ Exon27 leads to neuronal toxicity via gain of function caused by constitutive activation and increased motor association and aggregation.

\section{Results}

\section{KIF5A $\Delta$ Exon27 is aggregation prone}

We first expressed human wild type KIF5A (WT) and the ALS mutant $\triangle$ Exon27 with C-terminal GFP tags in HEK293T cells. 24 hours after transfection, WT mainly diffuses in the cytoplasm (Fig. 1B), consistent with previous reports (Kamata et al, 2017; Yoo et al, 2019). Interestingly, almost $100 \%$ of cells expressing $\Delta$ Exon27 show cytoplasmic granules of various sizes (Fig. 1B). At 48 hours, $4 \%$ WT expressing cells also showed a few granules, though significantly less abundant than those expressing $\Delta$ Exon27, which usually have one single large inclusion in the

101 cytoplasm (Fig. 1B). Similar results were also observed when these constructs were expressed in mouse neuroblastoma cells (N2a) (Fig. S1A). To exclude the possibility that this phenotype is 
103 due to the additional C-terminal GFP tag, we generated N-terminally FLAG-tagged KIF5A

104 constructs. Like the C-terminally GFP-tagged ones, the N-terminally FLAG-tagged $\Delta$ Exon27

105 also forms robust cytoplasmic granules in contrast to the homogeneously diffusive FLAG-tagged

106 WT (Fig. S1B).

107 To identify the properties of these granules, we first determined whether these granules are 108 positive for Ras GTPase-activating protein-binding protein 1 (G3BP1), a classical marker of 109 stress granules that are implicated in ALS pathogenesis (Li et al, 2013). Few stress granules were 110 observed in cells expressing either WT or $\Delta$ Exon27 (Fig. 1C, S1C) and merely $\sim 2 \% \Delta$ Exon27 111 granules co-stain with G3BP1 (Fig. S1D), indicating that $\Delta$ Exon27 granules are not stress 112 granules. Protein aggregations are characteristic features of many neurodegenerative diseases. 113 Because cytoplasmic inclusions of TAR DNA-binding protein 43 (TDP-43) were observed in $114>95 \%$ of ALS patients, (Neumann et al, 2006), we performed immunofluorescence staining to 115 detect TDP-43. In cells expressing either WT or $\triangle$ Exon27, TDP-43 remains within the nucleus 116 (Fig. S1E). However, we found that $\sim 83 \%$ of $\Delta$ Exon27 granules are positive for p62 and 117 ubiquitin, common components of cytoplasmic inclusions found in many protein aggregation 118 diseases (Fig. 1C, S1D). Immunoprecipitation of KIF5A using an antibody against the N119 terminal FLAG tag also pulls down endogenous p62 only in cells expressing $\Delta$ Exon27, but not 120 WT (Fig. S1F). We further determined the solubility of WT and $\Delta$ Exon27 in detergents of 121 different strength. In the RIPA-soluble fraction, the expression level of $\Delta$ Exon27 is comparable 122 to that of WT, suggesting that the propensity of $\Delta$ Exon27 to form cytoplasmic granules is not 123 due to its higher expression (Fig. 1D). In the RIPA-insoluble and urea-soluble fraction, we 124 detected significantly higher signal of $\Delta$ Exon27 compared to WT (Fig. 1E). These results 125 support that KIF5A $\triangle$ Exon27 with the altered C-terminal tail is aggregation prone.

129 KIF5A has been proposed to function as homodimers, with the dimerization domain located in 130 the stalk region (Kanai et al., 2000). Since ALS-associated KIF5A variants are autosomal 131 dominant, pathogenicity may be caused by mutant-WT KIF5A complexes. To mimic the patient 132 scenario and determine whether $\triangle$ Exon27 interacts with WT KIF5A, we co-expressed GFP133 tagged WT and mApple-tagged $\Delta$ Exon27 in HEK293T cells. WT and $\Delta$ Exon27 colocalize near- 
134 perfectly, forming robust cytoplasmic aggregates 48 hours after transfection (Fig. 2A). In 135 addition, immunoprecipitation of WT KIF5A pulls down $\triangle$ Exon27 when the two constructs are co-expressed (Fig. 2B). We also expressed $\triangle$ Exon27-GFP in N2a cells and stained for the

137 endogenous KIF5A WT using an antibody generated with a peptide corresponding to the C138 terminus of mouse KIF5A WT (amino acids 1007-1027), which does not recognize $\Delta$ Exon27.

139 While the endogenous KIF5A WT diffuses in the cytoplasm of non-transfected cells, it 140 colocalizes with $\Delta$ Exon27 aggregates in the transfected cells (Fig. 2C). These results suggest that 141 KIF5A $\triangle$ Exon27 interacts with WT and forms aggregates.

142 We further analyzed the expression pattern of WT and $\Delta$ Exon27 in primary mouse cortical 143 neurons. 24 hours after transfection, granules were observed in the soma of neurons expressing $144 \Delta$ Exon27 and were even more abundant along neurites and at the terminal end. In contrast, WT 145 KIF5A exhibits a diffuse pattern in the cytoplasm and along neurites (Fig. 3A). These granules 146 are positive for p62, again suggesting they are likely caused by motor aggregations (Fig. 3B).

147 We also co-stained these granules with the presynaptic marker, synapsin I, and observed neither 148 colocalization nor close proximity of the granules to synapses (Fig. S2A). Given the robust 149 accumulation of $\Delta$ Exon27 along the neurites and at the terminal ends, we hypothesized that $150 \Delta$ Exon 27 preferentially accumulates at the plus-ends of microtubules. To test this, we obtained a 151 highly polarized porcine kidney cell line (LLC-PK1) stably expressing tubulin-GFP (Rusan et al, 152 2001). Indeed, $\Delta$ Exon 27 granules were observed mainly at the peripheral extrusions (Fig. S2B) 153 and colocalizes with microtubule plus-end-tracking protein EB1 (Fig. S2C).

155 Protein inclusions formed by several disease-related proteins have been shown to trigger cellular 156 toxicity (Soto \& Pritzkow, 2018). To test whether aggregation-prone $\Delta$ Exon27 causes neuronal 157 toxicity, we co-transfected GFP, KIF5A WT-GFP, or $\triangle$ Exon27-GFP together with mApple in 158 mouse primary cortical neurons at DIV (days in vitro) 5 and used automated longitudinal 159 microscopy to track the survival of hundreds of neurons indicated by mApple fluorescence over 1606 days. The risk of death is significantly higher in neurons expressing $\triangle$ Exon27-GFP than those 161 expressing WT-GFP or GFP alone (Fig. 3C), indicating that $\triangle$ Exon27 is neurotoxic. enhanced processivity on microtubules 
166 The accumulation of $\Delta$ Exon27 granules at the plus-ends of microtubules suggests that the

167 localization of granules observed in distal neurites may be driven by aberrant motility and/or

168 association of the mutant protein along microtubules. Kinesin-1 motors transport cargoes by

169 converting chemical energy from ATP to mechanical energy to drive processive movement on

170 microtubules. When not transporting cargos, kinesin is autoinhibited to prevent ATP

171 squandering. Autoinhibition is achieved by the C-terminal tail domain binding to the motor

172 domain to keep it in a folded autoinhibited state (Dietrich et al, 2008; Kaan et al, 2011). Given

173 that $\Delta$ Exon27 possesses an altered C-terminal tail, we hypothesized that the autoinhibition of

174 KIF5A might be compromised. To test this, we performed single-molecule total internal

175 reflection fluorescence (smTIRF) microscopy assays to assess the mobility of three fluorophore-

176 tagged KIF5A proteins: WT, a KIF5A construct in which the C-terminal cargo domain was

177 removed rendering the motor incapable of autoinhibition (amino acids 1-906, referred to as $\Delta \mathrm{C}$,

178 Fig. S3A), and $\Delta$ Exon27. On microtubule filaments bound to the cover glass of the slide 179 chamber, KIF5A WT motors mostly show non-motile interactions with the microtubule (Fig.

180 4A), suggesting the majority of motors are primarily in the autoinhibited state. The few WT

181 motors that show motility display an average velocity of $0.45 \pm 0.11 \mu \mathrm{m} / \mathrm{s}$ and processivity of 0.6

$182[0.57,0.71] \mu \mathrm{m}$ (median with $95 \% \mathrm{CI}$ ). In both HEK293T and N2a cells, $\Delta \mathrm{C}$ also shows

183 diffused cytoplasmic expression like WT (Fig. S3B). As expected, and in contrast to WT, $\Delta \mathrm{C}$

184 exhibits enhanced motility and significantly increased velocity $(0.85 \pm 0.07 \mu \mathrm{m} / \mathrm{s})$ and

185 processivity $(1.63[1.47,1.81] \mu \mathrm{m})$ compared to WT as determined with the smTIRF assay (Fig.

186 4C, Table 1). Similar to $\Delta \mathrm{C}, \Delta$ Exon27 motors also exhibits enhanced motility events along

187 microtubules in the smTIRF assay, supporting the hypothesis that autoinhibition is relieved in

$188 \Delta$ Exon27. Intriguingly, while $\Delta$ Exon27 displays increased velocity $(0.66 \pm 0.10 \mu \mathrm{m} / \mathrm{s})$ compared

189 to WT, its velocity is slower than $\Delta \mathrm{C}$ and it exhibits drastically increased processivity (5.47

$190[3.84,7.80] \mu \mathrm{m})$ relative to $\Delta \mathrm{C}(p<0.0001$, Fig. 4B, Table 1). We also observed that the

191 fluorescence intensities of moving $\Delta$ Exon27 molecules were much stronger than those WT and

$192 \Delta \mathrm{C}$ (Fig. S4), indicating that $\Delta$ Exon27 promotes self-association (or aggregation) among KIF5A

193 motors and moves along the microtubules as multi-motor complexes. The recruitment of

194 additional motors would increase the number of motor domains near the microtubules thereby

195 decreasing the chance of complete dissociation of the motor complex from the microtubules 
196

during a run. Analysis of the initiation of motor movement indicates that $\Delta$ Exon 27 does not have a preference over the landing location on microtubules (Fig. S3C).

To determine whether the new $\mathrm{C}$-terminal tail of $\Delta$ Exon27 is required for the drastically increased processivity, we made a construct that contains the same sequence region between WT and $\Delta$ Exon27 (amino acids 1-998, referred to as K998, Fig. S3A). When expressed in HEK293T and N2a cells, K998 has diffused cytoplasmic localization as WT and $\Delta \mathrm{C}$ (Fig. S3B). In the smTIRF assay, K998 displays a similar velocity $(0.70 \pm 0.11 \mu \mathrm{m} / \mathrm{s})$ and processivity $(2.07$ [1.72, 2.50] $\mu \mathrm{m}$ ) as those observed for $\Delta \mathrm{C}$ (Fig. 4D, and Table 1). Importantly, the processivity of K998 is significantly lower than that of $\Delta$ Exon27 $(p<0.0001)$, and the fluorescence intensity of motile motors is similar to that of WT and $\Delta \mathrm{C}$ (Fig. S4). These results suggest that the new $\mathrm{C}$ terminal tail confers a gain-of-function via the relief of KIF5A autoinhibition and promotion of motor self-association, which in turn causes KIF5A $\Delta$ Exon27 motor complexes to move superprocessively along microtubules.

Finally, we characterized the motility of WT and $\Delta$ Exon27 complexes. WT and $\Delta$ Exon27, being fluorescently tagged by GFP and mApple, respectively, were co-expressed in HEK293T cell. In the presence of $\Delta$ Exon27, WT displays motile properties that resemble $\Delta$ Exon27. Analysis using either WT-GFP or $\triangle$ Exon27-mApple to assess motility yielded similar results for both velocity $(0.62 \pm 0.10 \mu \mathrm{m} / \mathrm{s}$ and $0.62 \pm 0.10 \mu \mathrm{m} / \mathrm{s}$, respectively) and processivity $(3.95[2.81,5.53] \mu \mathrm{m}$ and $4.49[3.64,5.63] \mu \mathrm{m}$, respectively) (Fig. 4E-F, S3D). Overlay of the kymograph of WT-GFP and $\Delta$ Exon27-mApple demonstrates that most of the moving spots consist of both WT and $\Delta$ Exon27 (Fig. S3D), consistent with our earlier observation that $\Delta$ Exon27 and WT form complexes with each other (Fig. 2).

\section{Ectopic expression of KIF5A $\Delta$ Exon27 in Drosophila leads to abnormal wings, motor deficits, paralysis, and premature death.}

To further investigate the role of KIF5A ALS mutant in vivo, we generated transgenic Drosophila expressing either WT or $\triangle$ Exon27 with a C-terminal GFP tag. To avoid any influence of the genomic environment on transgene expressions, a single copy of $U A S>h K I F 5 A$ transgenes were targeted to the well-established 68A4 landing site on chromosome III by PhiC31 
integrase mediated insertion. These transgenes are not expressed at baseline but will be transcribed when Gal4 is introduced by genetic cross. The protein levels of WT and $\Delta$ Exon27 are comparable when driven by the ubiquitously expressed tubulin-Gal4 (Fig. S6A). Constitutive ubiquitous expression of $\Delta$ Exon27 in Drosophila causes immature-looking adult escapers with unexpanded wings in $\sim 40 \%$ of flies and an early lethality (Fig. 5A-C). In contrast, neither the tubulin-Gal4 itself nor KIF5A WT causes any defects, confirming that the phenotype is

234 exclusively due to $\Delta$ Exon 27 expression.

Since ALS is a neuromuscular degenerative disease, we confined the expression of transgenes in fly muscles using an MHC-Gal4 driver. Contrary to KIF5A WT or MHC-Gal4, all the $\triangle$ Exon27expressing flies fully develop to the pupal stage as pharate adults but are unable to hatch from their pupal cases when raised at $28{ }^{\circ} \mathrm{C}$. After dissecting the $\Delta$ Exon27-expressing flies from their pupal case, we found that they have no obvious defects other than what could be expected from being paralyzed with folded wings and legs, wet appearance, and no movement (Fig. 5D). When raised at $25{ }^{\circ} \mathrm{C}, \Delta$ Exon27-expressing flies can develop to adults and have similar expression

243 levels as WT (Fig. S6B). However, they have dramatically reduced life span (Fig. S6C). We

244 further expressed KIF5A transgenes specifically in motor neurons using the commonly used 245 Drosophila motor neuron driver, C380-Gal4, and assessed their motor function with negative geotaxis assays. Flies expressing $\Delta$ Exon27 show impaired climbing activity compared to those expressing KIF5A WT or control flies expressing C380-Gal4 (Fig. 5E). Overall, our results highlight an imperative role of KIF5A $\triangle$ Exon 27 in mediating toxicity.

\section{Discussion}

Two different types of KIF5A variants in ALS patients were initially highlighted: a single nucleotide variant (SNV) caused by a missense mutation (rs11347976 [c.2957C>T]) that changes amino acid 986 from proline to leucine, and variants predicted to affect splicing of exon 27

255 (Brenner et al., 2018; Nicolas et al., 2018). Whether the identified SNV represents a disease256 causing or risk allele for ALS is unclear. Indeed, 11 of 29 patients carrying this SNV in the first 257 study also carry genetic mutations in other known ALS genes (Brenner et al., 2018). In contrast, 258 variants predicted to affect splicing of exon 27, although less common than rs11347976, are 259 highly penetrant and were subsequently identified in multiple patient cohorts around the world 
260 (Faruq et al., 2019; Nakamura et al., 2021; Naruse et al., 2021; Zhang et al., 2019). These 261 splicing variants were termed as "loss-of-function" mutations in the original studies since they 262 led to loss of exon 27 at the RNA level and the replacement of the normal C-terminal 34 amino 263 acids with a new tail of 39 amino acids at the protein level. However, the underlying disease 264 mechanism of KIF5A $\Delta$ Exon27 is currently unknown. Our results suggest that KIF5A $\Delta$ Exon27 265 causes toxicity mainly through gain of function. $\Delta$ Exon27 is particularly prone to form 266 cytoplasmic aggregates compared to WT or KIF5A with the deletion of C-terminal tails. In 267 addition, $\Delta$ Exon27 is relieved from autoinhibition and displays drastically increased processivity 268 through enhanced motor self-association caused by the altered C-terminal tail (Fig. 6). As a 269 result, un-inhibited motors bind to microtubules and move processively toward the microtubule 270 plus-ends and are therefore effectively removed from the pool of cytoplasmic motors needed for 271 plus-end-directed cargo transport. It has been shown that homozygous Kif5a knockout in mice is 272 embryonic lethal while heterozygous $\mathrm{Kif}^{+/-}$animals are completely normal, indicating that 273 having 50\% of normal KIF5A levels is sufficient for its normal physiological functions (Xia et $274 a l ., 2003)$. These results further support that KIF5A $\triangle$ Exon27 causes ALS through a toxic gain275 of-function mechanism.

277 In recent years, researchers have discovered that proteins bearing intrinsically disordered and 278 low complexity domains coalesce into liquid droplets, a phenotype called liquid-liquid phase 279 separation (LLPS). LLPS was initially recognized to play an important role in the formation and 280 function of membraneless organelles such as Cajal bodies, stress granules and nucleolus, to name 281 a few (Wang et al, 2021). It has also been shown to regulate local enrichment of molecules to 282 activate cell signaling and acceleration of biochemical reactions (Sheu-Gruttadauria \& MacRae, 283 2018; Su et al, 2016). By using seven different algorithms to predict proteins with intrinsic 284 disordered domains, Seeger et al. showed that intrinsically disordered residues are present 285 throughout the motor, stalk and tail domains of the kinesin family proteins. Strikingly, $71.8 \%$ 286 of kinesin tail domain residues are intrinsically disordered (Seeger \& Rice, 2013). When 287 expressed in mammalian cells, we found that KIF5A WT diffuses in the cytoplasm whereas $288 \Delta$ Exon27 forms small granules at an earlier time point, which eventually increase in size likely 289 by the fusion of multiple inclusions (Fig. 1B). It is interesting to note that similar but 290 significantly fewer granules were also observed for KIF5A WT with time and continuous protein 
accumulation. Although it has been proposed that KIF5A functions as homodimers, whether

292 KIF5A forms local condensates through LLPS and how this impact its role in transporting cargos

293 warrants further study. Importantly, dysfunctional LLPS of DNA- and RNA-binding proteins

294 such as TDP-43 and FUS have been implicated in ALS pathogenesis, leading to pathological

295 protein aggregates in patients (Pakravan et al, 2021). We showed that $\Delta$ Exon27 granules are

296 positive for p62 and ubiquitin and are only soluble in stringent detergent such as urea (Fig. 1C-

297 E, Fig. S1C-D). These data support that $\Delta$ Exon27 is prone to form cytoplasmic aggregates that

298 recruit p62- and ubiquitin-dependent proteolytic pathways.

299 Mutations of KIF5A have been previously linked to SPG10 and CMT2, two neurological

300 diseases with neuronal axonopathy (Crimella et al., 2012). While all the ALS-related KIF5A

301 variants are within the C-terminal tail, most SPG10/CMT2 mutations are located at the N-

302 terminal motor region with a few in the stalk domain. The unique positions of KIF5A mutations

303 that are associated with either SPG10/CMT2 or ALS provide an unprecedented opportunity to

304 delineate pathogenic mechanisms underlying ALS. The kinesin-1 family of motor proteins are

305 the best-understood members with respect to their mechanisms of motility at the single-

306 molecular level (Gennerich \& Vale, 2009). The fundamental regulatory mechanism for kinesin-1

307 is the transition from a "folded" (inhibited) to an "open" (activated) state (Fig. 6). In the folded

308 state, the tail/cargo-binding domain binds to the motor domain (or "head") and inhibits its

309 activity by blocking the release of ADP (autoinhibition) (Hackney et al, 2009; Seeger \& Rice,

310 2010). When the motor is activated presumably by cofactors or regulators, one of the two heads

311 releases ADP upon microtubule binding, which starts a productive processive run (Gennerich \&

312 Vale, 2009; Hancock, 2016). Using single-molecule assays, we found that KIF5A $\Delta$ Exon27

313 exhibits an increased velocity and a striking five-fold increase in processivity compared to

314 KIF5A WT (Fig. 4, Table 1). Surprisingly, the processivity of $\Delta$ Exon27 is much higher than $\Delta C$,

315 a construct where the C-terminal domain is deleted and autoinhibition is removed. This contrasts

316 with the previous studies on the motile properties of SPG10 mutants, which exhibited reduced

317 microtubule affinities and reduced velocities in microtubule-gliding assays (Ebbing et al, 2008).

318 In addition, KIF5A WT and $\triangle$ Exon27 interact to form oligomeric motors (Fig. 2) and display

319 increased velocity and processivity like $\Delta$ Exon27, suggesting that, when complexed, the mutant

320 KIF5A confer its gain of function property to the WT protein. The consequence of this

321 drastically increased processivity of KIF5A $\Delta$ Exon27 to neuronal health is currently unknown. In 
322 primary cortical neurons, $\Delta$ Exon 27 is highly accumulated along the neurites and at the neurite 323 terminals (Fig. 3A-B). Consistent with this, $\Delta$ Exon27 granules are mainly observed at the 324 peripheral extrusions of highly polarized LLC-PK1 cells and colocalize with microtubule plus325 end-tracking protein EB1 (Fig. S2B-C). These studies suggest that enhanced processivity may result in an aberrant distribution of the motor along the neurites, with an increase in the local concentration of $\Delta$ Exon 27 near the microtubule plus-ends, which in turn could increase motor self-association and aggregation. In addition, kinesin proteins need to be transported back to the cell body by dynein/dynactin-based retrograde transport after delivering cargos to distal ends. It is thus possible that an imbalance between the movement of antegrade and retrograde motor proteins will eventually disrupt intracellular transport. Importantly, we demonstrated that K998 with the truncated tail behaves more like $\Delta \mathrm{C}$ than $\Delta \mathrm{Exon} 27$. These results support the idea that 333 the new $\mathrm{C}$-terminal tail in $\Delta$ Exon27 is required to confer gain of toxicity through the relief of 334 autoinhibition and through self-association/aggregation of the mutant motors. In line with this notion, several single nucleotide deletion variants of KIF5A have also been identified in ALS patients (de Boer et al, 2021) which result in a C-terminal tail similar to that of the exon 27skiping variants: Variants c2987delA (p.Asp996fs), c.2989delA (p.Asn997fs), and c.2996delA (p.Asn999fs) cause one nucleotide frame shifting at amino acid positions Asp996, Asn997, and Asn999 at the protein level, respectively (Fig. S5).

The identification of ALS-associated KIF5A mutations adds KIF5A to a growing list of known

342 cytoskeletal-related genes implicated in ALS. We characterized $\Delta$ Exon27-mediated toxicity in a 343 transgenic Drosophila model. Drosophila is a versatile model system that has been widely used 344 in the ALS field to study the molecular mechanisms of key biological functions given their 345 genetic and overall experimental tractability (Liguori et al, 2021). We showed that ubiquitous 346 expression of $\Delta$ Exon27 in Drosophila cause wing abnormalities and shortened lifespan (Fig. 5A347 C). Tissue-specific expression revealed higher susceptibility to $\Delta$ Exon 27 expression in muscles 348 and led to paralysis (Fig. 5D). Motor neuron specific expression of $\Delta$ Exon 27 also impairs fly 349 climbing activities in negative geotaxis assays (Fig. 5E). The toxicity of $\Delta$ Exon27 is further 350 supported by observations that mouse cortical neurons expressing mutant KIF5A have reduced 351 survival (Fig. 3). Additional genetic studies for disease modifiers of toxicity using our 
Drosophila KIF5A ALS models will likely yield new mechanistic insights of disease pathogenesis and identify therapeutic strategies for this detrimental disease.

Materials and Methods

\section{DNA constructs}

358 Human KIF5A cDNA was a gift from Dr. Gary Bassell (Emory University) and was used as a template to generate the following GFP (EmGFP), mApple and FLAG tagged constructs: KIF5A-GFP, KIF5A-mApple, FLAG-KIF5A [full length wild type (WT), $\Delta$ Exon27, $\Delta$ cargo (amino acids 1-906, $\Delta$ C), K998 (amino acids 1-998)]. Constructs used for in vitro studies were cloned into the pGW1-EmGFP backbone (a gift from Dr. Sami Bermada, University of Michigan) by PCR based cloning (Invitrogen). For longitudinal fluorescence microscopy pGW1mApple was used. The human KIF5A WT and $\triangle$ Exon27 were subcloned to the pUAST-attB backbone, a gift from Dr. Ken Moberg (Emory University), for the generation of transgenic Drosophila.

\section{Drosophila stocks and genetics}

Fly crosses were maintained at $25^{\circ} \mathrm{C}$ in a humidified chamber with 12-hour light-dark cycles. Fly food was prepared with a standard recipe (water; cornmeal; yeast; agar; molasses; propionic acid). Parental stocks were maintained at room temperature. Transgenic flies $U A S>h K I F 5 A W T$ GFP and $U A S>h K I F 5 A \triangle E x o n 27-G F P$ were generated by inserting the respective transgenes

373 into the attP2 site of $y^{1} w^{67 c 23}$; P[CaryP]attP2 (BI \#8622) by injection and phiC31 integration

374 (BestGene Inc, USA). The tubulin-gal4 and MHC-Gal4 line were generously provided by Dr. 375 Ken Moberg (Emory University) and Dr. Udai Pandey (University of Pittsburgh), respectively.

376 The motor neuron driver line C380-Gal4 was purchased from Bloomington Drosophila Stock

377 Center (BI \# 80580). Flies were imaged using a Leica MC170 HD digital camera mounted on a 378 Nikon SMZ800N stereo microscope.

380 Drosophila survival assay

381 For the survival assay, parental flies were raised with standard food and under 12-hour day/night 382 cycles at $25^{\circ} \mathrm{C}$ unless otherwise stated. The parents were allowed to mate and lay eggs for 3 days 383 before discarded. The offspring from these parents were collected over a period of 24 hours and 
sorted by sex. Up to 12 male flies were kept in individual vials containing standard food. For each genotype, multiple replicate vials were set up so that the total sample size was 75 for each genotype. Flies were transferred onto fresh food every 2-3 days. The number of deaths was recorded each day. All survival assays were performed at $25^{\circ} \mathrm{C}$.

\section{Negative geotaxis assay}

390 Fly climbing activity was assessed using negative geotaxis assay as previously described 391 (Nichols et al, 2012). Briefly, 15 flies were transferred, without anesthetization, into each plastic 392 vial and placed in the apparatus. The vials were tapped down against the bench and the climbing 393 was recorded on video for 1 minutes. The percent of flies climbing to the top of vials in 30 s was 394 determined manually in a blinded manner.

\section{Cell culture and transfection}

397 Human embryonic kidney (HEK293T), and mouse neuroblastoma (N2a) cell lines from ATCC.

398 The porcine kidney cell line (LLC-PK1) was a gift from Dr. Melissa Gardner (University of 399 Minnesota). Cells were cultured in high glucose DMEM (Invitrogen) supplemented with 10\%

400 fetal bovine serum (Corning), $4 \mathrm{mM}$ Glutamax (Invitrogen), penicillin (100 U/mL), streptomycin $401(100 \mu \mathrm{g} / \mathrm{mL})$ and non-essential amino acids (1\%). Cells were grown at $37^{\circ} \mathrm{C}$ in a humidified 402 atmosphere with $5 \% \mathrm{CO}_{2}$. Cells were transiently transfected using polyethylenimine $(1 \mathrm{mg} / \mathrm{mL})$ 403 or Lipofectamine 2000 (Invitrogen). Experiments were performed either 24 or 48 hours after 404 transfection.

\section{Primary cortical neuronal culture and transfection}

407 Primary cortical neurons were prepared from C57BL/6J mouse embryos (Charles River) of either 408 sex on embryonic day 17. Cerebral cortices were dissected and enzymatically dissociated using 409 trypsin w/ EDTA (Thermo Fisher Scientific; 10 minutes), mechanically dissociated in Minimum 410 Essential Media (MEM; Fisher) supplemented with 0.6\% glucose (Sigma) and 10\% Fetal Bovine 411 Serum (FBS; Hyclone). Neurons were plated on coverslips (Matsunami Inc., $22 \mathrm{~mm}$ ) or MatTek 412 dishes coated with poly-l-lysine (Sigma). A total of 50,000 neurons were plated as a 'spot' on the 413 center of the coverslip to create a small, high-density network. Neurons were cultured in standard 414 growth medium [glial conditioned neurobasal plus medium (Fisher) supplemented with 415 Glutamax (GIBCO) and B27 plus (Invitrogen)], and half of the media was exchanged 2-3 times a 
416 week until the experiment endpoints. No antibiotics or antimycotics were used. Cultures were

417 maintained in an incubator regulated at $37{ }^{\circ} \mathrm{C}, 5 \% \mathrm{CO}_{2}$ and $95 \%$ relative humidity as described

418 (Valdez-Sinon et al, 2020). Cells were transiently transfected using Lipofectamine 2000

419 (Invitrogen) according to the manufacturer's instructions.

\section{Longitudinal fluorescence microscopy}

422 Mouse primary cortical neurons were transfected with mApple and various KIF5A constructs 423 and imaged by fluorescence microscopy at 24 hour intervals for 7 days as described (Weskamp 424 et al, 2019). Custom scripts were used to automatically generate region of interest corresponding 425 to each cell and determine time of death based on rounding of the soma, retraction of neurites, or 426 loss of fluorescence. The time of death for individual neurons was used to calculate the risk of 427 death in each population relative to a reference group. Images were acquired using Keyence BZ428 X810 microscope with a 10× objective and analyzed by Image J. The images were stitched and 429 stacked, and cell death was scored using the criteria mentioned above.

\section{Immunofluorescence}

432 Cells were fixed in 4\% paraformaldehyde (Electron Microscopy Sciences) for 20 minutes, 433 washed three times for 5 minutes with phosphate buffer saline (1× PBS, Corning) and treated 434 with $0.1 \%$ Triton $\times-100$ (Sigma) in PBS. Cells were blocked for 30 minutes in blocking solution 435 consisting of $4 \%$ bovine serum albumin (Sigma) in PBS. Cells were incubated overnight in 436 primary antibodies: rabbit anti-G3BP1 (Proteintech), rabbit anti-p62 (Proteintech), mouse anti437 p62 (Novus Biologicals), rabbit anti-TDP-43 (Proteintech), rabbit anti-synapsin I (Sigma), 438 guinea pig anti-MAP2 (Synaptic Systems) diluted in blocking solution (1:500). The next day, 439 cells were washed 3 times for 5 minutes in PBS and incubated in secondary antibodies in 440 blocking solution for one hour at room temperature. After washing 3 times for 5 minutes, 441 coverslips with the cells were mounted using Prolong Gold Antifade mounting media 442 (Invitrogen). Images were acquired with Keyence BZ-X810 microscope with a 60× oil objective. 443 For image analysis, around 100-150 transfected cells were counted for each genotype in each 444 experiment. Quantification of colocalization of $\Delta$ Exon27 aggregates with G3BP1, p62, and 445 ubiquitin were performed manually in a blinded manner using ImageJ analysis. 
448 Whole cell extracts were isolated using RIPA Lysis Buffer pH 7.4 (Bio-world, USA) 449 supplemented with Halt ${ }^{\mathrm{TM}}$ protease and phosphatase inhibitor cocktail (ThermoFisher Scientific),

450 followed by DNA shearing. After centrifuge, the pellet was washed for 3 times and dissolved in $4518 \mathrm{M}$ urea buffer (10 mM Tris, $\mathrm{pH} 8.0 ; 8 \mathrm{M}$ urea). The concentration of the isolated proteins was 452 determined using BCA Protein Assay Reagent (Pierce, USA). $20 \mu \mathrm{g}$ of protein was resolved in 453 4-20\% precast polyacrylamide gel (Bio-Rad, USA). For immunoprecipitation, HEK 293T cells 454 were collected and lysed using Pierce ${ }^{\mathrm{TM}}$ IP Lysis Buffer (ThermoFisher Scientific) supplemented 455 with Halt ${ }^{\mathrm{TM}}$ protease and phosphatase inhibitor cocktail (ThermoFisher Scientific), and $500 \mu \mathrm{g}$ 456 of protein lysate was precleared with $25 \mu 1$ of Chromotek GFP-Trap ${ }^{\circledR}$ or Pierce ${ }^{\mathrm{TM}}$ Anti-FLAG 457 magnetic agarose beads. Following the manufacturer's instructions, protein lysate was 458 immunoprecipitated for $2 \mathrm{~h}$ at $4^{\circ} \mathrm{C}$ with the following antibodies: mouse anti-GFP (1:1000; 459 Takara Bio), mouse anti-FLAG (1:1000; Sigma). The input 10\% was analyzed with Western 460 blot. Proteins were transferred to nitrocellulose membranes $(0.2 \mu \mathrm{m}$, Bio-Rad $)$ and incubated 461 with primary antibodies: mouse anti-GFP (1:2000; Clontech), mouse anti-FLAG (1:1000; 462 Sigma), rabbit anti-KIF5A N-terminus (1:1000; GeneTex), rabbit anti-KIF5A C-terminus 463 (1:1000; abcam), rabbit anti- $\beta$ actin $\left(1: 2000\right.$; GeneTex) overnight at $4^{\circ} \mathrm{C}$ followed by HRP464 conjugated secondary antibodies (ABclonal) or IRDye secondary antibodies (Li-cor) at room 465 temperature for 1 hour. Super Signal West Pico (Pierce, USA) was used for detection of 466 peroxidase activity. Molecular masses were determined by comparison to protein standards 467 (Thermo Scientific). Band intensities were measured using ImageJ and normalized to tubulin or 468 actin.

\section{Generation, expression, and purification of K490-EmGFP}

471 K490-EmGFP gene fragment was generated by PCR with 25 bp overlap with a modified NEB 472 SNAP-tag backbone in which the SNAP-tag was replaced by a C-term 6His tag. The plasmid 473 was generated by Gibson assembly. The insertion was confirmed by enzymatic digestion and 474 agarose gel electrophoresis. Protein expression and purification were performed as previously 475 described (Budaitis et al, 2021). Briefly, the plasmid was transformed into BL21-CodonPlus 476 (DE3)-RIPL competent cells (Agilent, \#230280). A single colony was inoculated in 1-mL TB 477 medium (Laboratory, 2006) with $50 \mu \mathrm{g} / \mathrm{mL}$ chloramphenicol and $25 \mu \mathrm{g} / \mathrm{mL}$ carbenicillin. After 478 shaken at $37{ }^{\circ} \mathrm{C}$ overnight, the $1 \mathrm{~mL}$ culture was inoculated into $400 \mathrm{~mL} \mathrm{~TB}$ medium and was 
shaken at $37^{\circ} \mathrm{C}$ for 5 hours. The cell culture was then cool down on ice to $<18^{\circ} \mathrm{C}$, and IPTG was added to final $0.1 \mathrm{mM}$ concentration. The protein expression was induced at $18^{\circ} \mathrm{C}$ overnight with vigorous shaking. Afterwards, the cell was harvested by centrifugation at $3000 \mathrm{~g}$ for $10 \mathrm{~min}$. The supernatant was discarded, and the pellet was resuspended in $5 \mathrm{~mL}$ B-PER ${ }^{\mathrm{TM}}$ complete bacterial protein extraction reagent (Thermo Scientific, \#89821) supplemented with $2 \mathrm{mM}$ DTT, $0.2 \mathrm{mM}$ ATP, $4 \mathrm{mM} \mathrm{MgCl}_{2}, 2 \mathrm{mM}$ EGTA, and $2 \mathrm{mM}$ PMSF. The resuspended solution was flash frozen and stored at $-80{ }^{\circ} \mathrm{C}$ before purification.

To purify K490-EmGFP, the cell solution was thawed at $37{ }^{\circ} \mathrm{C}$, followed by nutation at room temperature for $20 \mathrm{~min}$. The cells were further lysed via douncing for 10 strokes on ice, and then cleared by centrifuging at $80000 \mathrm{rpm}$ for $10 \mathrm{~min}$ at $4{ }^{\circ} \mathrm{C}$ using a Beckman Coulter tabletop centrifuge. At the same time, $0.5 \mathrm{~mL}$ Roche Ni-NTA resin (MilliporeSigma, \#5893682001) was washed with $2 \times 1 \mathrm{~mL}$ of wash buffer [50 mM HEPES, $300 \mathrm{mM} \mathrm{KCl,} 2 \mathrm{mM} \mathrm{MgCl} 2,1 \mathrm{mM}$ EGTA, $10 \%$ glycerol, 1 mM DTT, 0.1 mM ATP, 1 mM PMSF, $0.1 \%$ Pluronic F-127 (w/v), pH 7.2]. The cleared lysate was carefully added to the Ni-NTA resin and allowed to flow through. The resin was then washed with $5 \times 2 \mathrm{~mL}$ wash buffer. The protein was eluted with elution buffer [50 mM HEPES (pH 7.2), 150 mM KCl, 2 mM MgCl 2,1 mM EGTA, 250 mM imidazole (pH

\section{Microtubule polymerization}

Microtubule polymerization was performed as described (Rao et al, 2018). Briefly, $2 \mu \mathrm{L}$ of 1 $\mathrm{mg} / \mathrm{mL}$ Cy5-labeled tubulin (prepared as described in (Nicholas et al, 2014)), $2 \mu \mathrm{L}$ of $1 \mathrm{mg} / \mathrm{mL}$ biotinylated tubulin (Cytoskeleton Inc., \#T333P), and $2 \mu \mathrm{L}$ of $10 \mathrm{mg} / \mathrm{mL}$ unlabeled tubulin (Cytoskeleton Inc., \#T240) was mixed on ice. $0.6 \mu \mathrm{L}$ of $10 \mathrm{mM}$ GTP was added to the tubulin solution, and the mixture was incubated at $37{ }^{\circ} \mathrm{C}$ for 15 minutes. Then $0.72 \mu \mathrm{L}$ of $0.1 \mathrm{mM}$ taxol in DMSO was added to the mixture, which was further incubated at $37{ }^{\circ} \mathrm{C}$ for 15 minutes. Tubulin that was not incorporated into the microtubules were removed by centrifugation through a glycerol cushion [BRB80 (80 mM PIPES, $2 \mathrm{mM} \mathrm{MgCl} 2,1 \mathrm{mM}$ EGTA) with 60\% (v/v) glycerol, $10 \mu \mathrm{M}$ taxol, and $1 \mathrm{mM}$ DTT, $\mathrm{pH}$ 6.8]. The pellet was re-suspended in resuspension 507 buffer [BRB80 with 10\% (v/v) glycerol, $10 \mu \mathrm{M}$ taxol, and $1 \mathrm{mM}$ DTT, pH 6.8] to final 508 concentration of $1 \mathrm{mg} / \mathrm{mL}$ microtubules. The microtubule solution was stored at room temperature in the dark for several days. 


\section{Single-molecule total internal reflection fluorescence (smTIRF) microscopy assay} transfection, cells were washed with sterile PBS and detached from the dish using a cell scraper.

514 Cells were resuspended in ice cold RIPA lysis buffer supplemented with Halt ${ }^{\mathrm{TM}}$ protease and 515 phosphatase inhibitor cocktail (Thermo Fisher Scientific). Lysates were centrifuged at 17,000 $g$ 516 for 5 minutes at $4{ }^{\circ} \mathrm{C}$. The supernatant was aliquoted, flashed frozen in liquid nitrogen, and 517 stored at $-80{ }^{\circ} \mathrm{C}$. smTIRF assay was carried out similarly as described before (Rao et al., 2018). 518 Briefly, a coverslip (No. 1.5, Zeiss, \#474030-9000-000) was cleaned by ethanol, and a flow 519 chamber was assembled using the cleaned coverslip, a glass slide, and two stripes of cut parafilm 520 as described before (Nicholas et al., 2014). $0.5 \mathrm{mg} / \mathrm{mL}$ of BSA-biotin was flown into the 521 chamber, and the slide was incubated in a humidity box for 5 minutes. Afterwards, the chamber 522 was washed with $3 \times 20 \mu \mathrm{L}$ of blocking buffer (BB) [BRB80 with 1\% Pluronic F-127 (w/v), 10

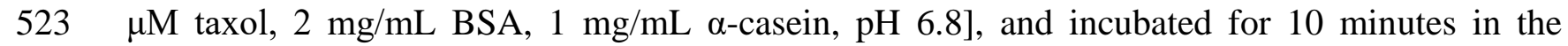
524 humidity box. After the surface was passivated, $0.25 \mathrm{mg} / \mathrm{mL}$ of streptavidin was flown into the 525 chamber and incubated for 5 minutes. The chamber was then washed with $3 \times 20 \mu \mathrm{L}$ BB. $0.5 \mu \mathrm{L}$ 526 of $0.2 \mathrm{mg} / \mathrm{mL}$ microtubules was diluted in $19.5 \mu \mathrm{L} \mathrm{BB}$ and subsequently flown through the 527 chamber. The chamber was washed with $2 \times 20 \mu \mathrm{L} \mathrm{BB}$ and $20 \mu \mathrm{L}$ motility buffer (MB) [60 mM 528 HEPES, $50 \mathrm{mM} \mathrm{KAc,} \mathrm{mM} \mathrm{MgCl}_{2}, 1 \mathrm{mM}$ EGTA, 0.5\% Pluronic F-127 (w/v), $10 \%$ glycerol (v/v), $52910 \mu \mathrm{M}$ taxol, $1 \mathrm{mM}$ DTT, $2 \mathrm{mg} / \mathrm{mL}$ BSA, $1 \mathrm{mg} / \mathrm{mL} \alpha$-casein, $\mathrm{pH}$ 7.2]. The final assay solution 530 was prepared by mixing $3 \mu \mathrm{L}$ of $50 \mathrm{mg} / \mathrm{mL}$ BSA, $1 \mu \mathrm{L}$ of $100 \mathrm{mM}$ ATP, $2 \mu \mathrm{L}$ of $25 \times$ protease 531 inhibitor cocktail (Roche, \#4693159001), $1 \mu \mathrm{L}$ of $50 \mathrm{mM}$ biotin, $1 \mu \mathrm{L}$ of appropriated diluted 532 HEK293T cell lysate or K490, and $42 \mu \mathrm{L}$ of MB. The final dilution of the lysate was $200 \times$ to $5331000 \times$ depending on the construct. The final dilution of K490 was $10000 \times(200 \mathrm{pM}) .2 \times 20 \mu \mathrm{L}$ 534 of the assay solution was flown through the chamber, and the chamber was sealed by vacuum 535 grease. The images were acquired by $200 \mathrm{~ms} /$ frame. The data was analyzed using a home-built 536 MATLAB program.

539 Statistical analyses and graphs were prepared in GraphPad Prism (version 9). Data are expressed 540 as mean \pm SD or mean \pm SEM as shown in figure legends. Student t-test or one-way ANOVA 
was used for statistical analysis unless specified in figure legends. $p$ less than 0.05 was considered significant $(* p<0.05, * * p<0.01, * * * p<0.001)$.

\section{References}

Blair MA, Ma S, Hedera P (2006) Mutation in KIF5A can also cause adult-onset hereditary spastic paraplegia. Neurogenetics 7: 47-50

550 Brenner D, Yilmaz R, Muller K, Grehl T, Petri S, Meyer T, Grosskreutz J, Weydt P, Ruf W, 551 Neuwirth C et al (2018) Hot-spot KIF5A mutations cause familial ALS. Brain 141: 688-697 Budaitis BG, Jariwala S, Rao L, Yue Y, Sept D, Verhey KJ, Gennerich A (2021) Pathogenic mutations in the kinesin-3 motor KIF1A diminish force generation and movement through allosteric mechanisms. J Cell Biol 220

Chia R, Chio A, Traynor BJ (2018) Novel genes associated with amyotrophic lateral sclerosis: diagnostic and clinical implications. Lancet Neurol 17: 94-102 Crimella C, Baschirotto C, Arnoldi A, Tonelli A, Tenderini E, Airoldi G, Martinuzzi A, Trabacca A, Losito L, Scarlato M et al (2012) Mutations in the motor and stalk domains of KIF5A in spastic paraplegia type 10 and in axonal Charcot-Marie-Tooth type 2. Clin Genet 82: 157-164 de Boer EMJ, van Rheenen W, Goedee HS, Kamsteeg EJ, Brilstra EH, Veldink JH, van Den Berg LH, van Es MA (2021) Genotype-phenotype correlations of KIF5A stalk domain variants. Amyotrophic lateral sclerosis \& frontotemporal degeneration: 1-10

Dietrich KA, Sindelar CV, Brewer PD, Downing KH, Cremo CR, Rice SE (2008) The kinesin-1 motor protein is regulated by a direct interaction of its head and tail. Proc Natl Acad Sci U S A 105: 8938-8943

Duis J, Dean S, Applegate C, Harper A, Xiao R, He W, Dollar JD, Sun LR, Waberski MB, Crawford TO et al (2016) KIF5A mutations cause an infantile onset phenotype including severe myoclonus with evidence of mitochondrial dysfunction. Ann Neurol 80: 633-637

Ebbing B, Mann K, Starosta A, Jaud J, Schols L, Schule R, Woehlke G (2008) Effect of spastic paraplegia mutations in KIF5A kinesin on transport activity. Hum Mol Genet 17: 1245-1252 Faruq M, Kumar D, Wadhwa S, Shamim U, Mathur A, Parveen S, Garg A, Srivastava AK (2019) Intrafamilial variable spastic paraplegia/ataxia/ALS phenotype linked to a novel KIF5A mutation. Clin Genet 96: 271-273 Fichera M, Lo Giudice M, Falco M, Sturnio M, Amata S, Calabrese O, Bigoni S, Calzolari E, Neri M (2004) Evidence of kinesin heavy chain (KIF5A) involvement in pure hereditary spastic paraplegia. Neurology 63: 1108-1110

578 Gennerich A, Vale RD (2009) Walking the walk: how kinesin and dynein coordinate their steps. 579 Curr Opin Cell Biol 21: 59-67

580 Hackney DD, Baek N, Snyder AC (2009) Half-site inhibition of dimeric kinesin head domains by monomeric tail domains. Biochemistry 48: 3448-3456

584 Hirokawa N, Noda Y, Tanaka Y, Niwa S (2009) Kinesin superfamily motor proteins and 585 intracellular transport. Nat Rev Mol Cell Biol 10: 682-696 
Kaan HY, Hackney DD, Kozielski F (2011) The structure of the kinesin-1 motor-tail complex reveals the mechanism of autoinhibition. Science 333: 883-885 Kamata H, Tsukasaki Y, Sakai T, Ikebe R, Wang J, Jeffers A, Boren J, Owens S, Suzuki T, Higashihara $\mathrm{M}$ et al (2017) KIF5A transports collagen vesicles of myofibroblasts during pleural fibrosis. Sci Rep 7: 4556

591 Kanai Y, Okada Y, Tanaka Y, Harada A, Terada S, Hirokawa N (2000) KIF5C, a novel neuronal kinesin enriched in motor neurons. J Neurosci 20: 6374-6384 Laboratory CSH (2006) Terrific Broth. Cold Spring Harb Protoc pdb.rec8620 Li YR, King OD, Shorter J, Gitler AD (2013) Stress granules as crucibles of ALS pathogenesis. The Journal of cell biology 201: 361-372 Liguori F, Amadio S, Volonte C (2021) Fly for ALS: Drosophila modeling on the route to amyotrophic lateral sclerosis modifiers. Cell Mol Life Sci 78: 6143-6160 Nakamura R, Tohnai G, Atsuta N, Nakatochi M, Hayashi N, Watanabe H, Yokoi D, Watanabe H, Katsuno M, Izumi Y et al (2021) Genetic and functional analysis of KIF5A variants in Japanese patients with sporadic amyotrophic lateral sclerosis. Neurobiol Aging 97: 147 e111-147 e117 Naruse H, Ishiura H, Mitsui J, Takahashi Y, Matsukawa T, Sakuishi K, Nakamagoe K, Miyake Z, Tamaoka A, Goto J et al (2021) Splice-site mutations in KIF5A in the Japanese case series of amyotrophic lateral sclerosis. Neurogenetics 22: 11-17

Neumann M, Sampathu DM, Kwong LK, Truax AC, Micsenyi MC, Chou TT, Bruce J, Schuck T, Grossman M, Clark CM et al (2006) Ubiquitinated TDP-43 in frontotemporal lobar degeneration and amyotrophic lateral sclerosis. Science (New York, NY) 314: 130-133 Nicholas MP, Rao L, Gennerich A (2014) Covalent immobilization of microtubules on glass surfaces for molecular motor force measurements and other single-molecule assays. Methods Mol Biol 1136: 137-169

Nichols CD, Becnel J, Pandey UB (2012) Methods to assay Drosophila behavior. Journal of visualized experiments : JoVE

Nicolas A, Kenna KP, Renton AE, Ticozzi N, Faghri F, Chia R, Dominov JA, Kenna BJ, Nalls MA, Keagle P et al (2018) Genome-wide Analyses Identify KIF5A as a Novel ALS Gene.

Neuron 97: 1268-1283 e1266

Pakravan D, Orlando G, Bercier V, Van Den Bosch L (2021) Role and therapeutic potential of liquid-liquid phase separation in amyotrophic lateral sclerosis. J Mol Cell Biol 13: 15-28 Rao L, Hulsemann M, Gennerich A (2018) Combining Structure-Function and Single-Molecule Studies on Cytoplasmic Dynein. Methods Mol Biol 1665: 53-89

Rusan NM, Fagerstrom CJ, Yvon AM, Wadsworth P (2001) Cell cycle-dependent changes in microtubule dynamics in living cells expressing green fluorescent protein-alpha tubulin. Mol Biol Cell 12: 971-980

Rydzanicz M, Jagla M, Kosinska J, Tomasik T, Sobczak A, Pollak A, Herman-Sucharska I, Walczak A, Kwinta P, Ploski R (2017) KIF5A de novo mutation associated with myoclonic seizures and neonatal onset progressive leukoencephalopathy. Clin Genet 91: 769-773

Seeger MA, Rice SE (2010) Microtubule-associated protein-like binding of the kinesin-1 tail to microtubules. J Biol Chem 285: 8155-8162

Seeger MA, Rice SE (2013) Intrinsic Disorder in the Kinesin Superfamily. Biophys Rev 5

630 Human miRISC. Cell 173: 946-957 e916 
631 Soto C, Pritzkow S (2018) Protein misfolding, aggregation, and conformational strains in

632 neurodegenerative diseases. Nat Neurosci 21: 1332-1340

633 Su X, Ditlev JA, Hui E, Xing W, Banjade S, Okrut J, King DS, Taunton J, Rosen MK, Vale RD

634 (2016) Phase separation of signaling molecules promotes T cell receptor signal transduction.

635 Science 352: 595-599

636 Tanaka Y, Kanai Y, Okada Y, Nonaka S, Takeda S, Harada A, Hirokawa N (1998) Targeted

637 disruption of mouse conventional kinesin heavy chain, kif5B, results in abnormal perinuclear

638 clustering of mitochondria. Cell 93: 1147-1158

639 Valdez-Sinon AN, Lai A, Shi L, Lancaster CL, Gokhale A, Faundez V, Bassell GJ (2020) Cdh1-

640 APC Regulates Protein Synthesis and Stress Granules in Neurons through an FMRP-Dependent

641 Mechanism. iScience 23: 101132

642 Wang B, Zhang L, Dai T, Qin Z, Lu H, Zhang L, Zhou F (2021) Liquid-liquid phase separation

643 in human health and diseases. Signal Transduct Target Ther 6: 290

644 Weskamp K, Safren N, Miguez R, Barmada S (2019) Monitoring Neuronal Survival via

645 Longitudinal Fluorescence Microscopy. J Vis Exp

646 Xia CH, Roberts EA, Her LS, Liu X, Williams DS, Cleveland DW, Goldstein LS (2003)

647 Abnormal neurofilament transport caused by targeted disruption of neuronal kinesin heavy chain

648 KIF5A. J Cell Biol 161: 55-66

649 Yoo KS, Lee K, Oh JY, Lee H, Park H, Park YS, Kim HK (2019) Postsynaptic density protein

65095 (PSD-95) is transported by KIF5 to dendritic regions. Mol Brain 12: 97

651 Zhang K, Liu Q, Shen D, Tai H, Liu S, Wang Z, Shi J, Fu H, Wu S, Ding Q et al (2019)

652 Mutation analysis of KIF5A in Chinese amyotrophic lateral sclerosis patients. Neurobiol Aging

653 73: 229 e221-229 e224

654

655

656

Acknowledgements: We thank members of the Jiang and Bassell labs for their helpful

657 technical help and discussions.

658

659 Funding: JP is supported by the Milton Safenowitz Postdoctoral Fellowship from the ALS

660 association.

661 LR and AG are supported by National Institutes of Health (NIH) grants R01GM098469 and

662 R01NS114636.

663 LS and GJB are supported by the NIH R01 (R01NS114253 to GJB).

664 The work is partially funded by the NIH R01 grant R01AG068247 to JJ.

665

666 Author contributions: DCP, JP, and JJ performed and analyzed all in vivo and cell-based

667 experiments. LR performed and analyzed the single-molecule motility assays. AG designed and

668 interpreted single-molecule data and edited the manuscript. GC helped in data analysis and 
Drosophila experiments. ZTM and JG provided biological reagents. DCP, JP, and JJ wrote the manuscript.

Competing interests: All other authors declare they have no competing interests.

Data and materials availability: All data are available in the main text or the supplementary materials.

Figures and tables

Fig. 1. ALS-associated KIF5A $\Delta$ Exon27 is prone to form cytoplasmic aggregates. domains (left). ALS-associated KIF5A variants disrupt the C-terminal tail by skipping exon 27 during mRNA splicing and produce a new C-terminal tail of 39 amino acids (red) replacing the normal tail of 34 amino acids (right). (B) HEK293T cells were transfected with KIF5A WT-GFP

693 G3BP1, p62, and Ubiquitin with KIF5A $\triangle$ Exon27 granules. (D-E) Western blot analysis of 694 RIPA soluble-fraction (D) and RIPA-insoluble, urea-soluble fraction of proteins prepared from 695 HEK293T cells expressing empty plasmid (control), FLAG-KIF5A WT, FLAG- $\Delta$ Exon27. 696 Insoluble/soluble KIF5A fractions were detected with anti-FLAG and actin was used as a 697 loading control. Data represent the mean \pm SD.

Fig. 2. $\Delta$ Exon27 interacts with WT KIF5A to form aggregates

(A) Expression of KIF5A WT and $\triangle$ Exon27 tagged with GFP and mApple, respectively, in 701 HEK293T cells. Images were taken 48 hours after transfection. (B) Pull down analysis showing 702 the association of $\triangle$ Exon27 with WT KIF5A. (C) Staining of mouse endogenous KIF5A in N2a 703 cells transfected with $\triangle$ Exon27-GFP using an antibody generated with a peptide corresponding 
704 to the C-terminus of KIF5A WT (amino acids 1007-1027), which does recognize $\Delta$ Exon27.

$705 \Delta$ Exon27 granules are highlighted with arrowheads.

Fig. 3. KIF5A $\Delta$ Exon 27 causes neuronal toxicity.

708 (A) Mouse primary neurons transfected with KIF5A WT-GFP and $\triangle$ Exon27-GFP were probed 709 using anti-GFP antibody 24 hours after transfection. Neurons were identified by microtubule 710 associated protein 2 (MAP2) and nuclei were stained with DAPI. (B) p62 staining in neurons 711 expressing $\Delta$ Exon27-GFP. Colocalization of p62 with $\Delta$ Exon27 granules are highlighted with 712 arrow heads. (C) 5-day-old mouse cortical neurons were transfected with mApple together with 713 either GFP, KIF5A WT-GFP, or $\triangle$ Exon27-GFP. Images were taken every 24 hours after 714 transfection. Survival of neurons was analyzed by Kaplan-Meier survival analysis $(* * * p<$ $7150.0001, \log$-rank test); 3 individual experiments were performed with similar results.

716 Fig. 4. KIF5A $\Delta$ Exon27 relieves motor autoinhibition and displays increased velocity and 717 processivity.

718 Kymographs and motility analyses of non-motile and mobile KIF5A proteins prepared from 719 HEK293T cells. (A) KIF5A WT-GFP. From kymographs (example on the left), single-motor 720 velocities (middle) and run lengths (right) were determined. (B) As in $A$, but for $\triangle$ Exon27-GFP. 721 (C) As in $A$, but for $\triangle \mathrm{C}$-GFP; (D) As in $A$, but for K998-GFP. The X-axis scale for the 722 processivity graphs was limited to $20 \mu \mathrm{m}$ to permit a direct comparison of the run lengths of the 723 different constructs. Diagonal lines in the kymograph represent KIF5A molecules moving over 724 time. The depicted scale bars are the same for all kymographs shown in this figure. The velocity 725 data were fit with Gaussian distribution and the processivity data were fit with an exponential 726 decay function. (E-F) Statistical analysis of the velocity (E) and processivity (F) of various 727 KIF5A proteins. The green bars represent the median with 95\% CI. The statistical comparison of 728 velocity was performed using unpaired parametric t-test $(* * * p<0.001)$. The run length is in log 729 scale. The statistical evaluation of processivity was performed using ANOVA two way $(* * * p<$ 730 0.001). The measured values for the velocities and run lengths are listed in Table 1.

732 Fig. 5. KIF5A $\Delta$ Exon27 is toxic in Drosophila melanogaster.

733 (A) Ubiquitous overexpression of $\Delta$ Exon27 leads to wing defects (black arrows). (B) Percent of 734 flies with normal and abnormal wings ( $\mathrm{n}=50$ flies, 2 biological repeats). (C) Life spans were 
735 analyzed for flies expressing KIF5A WT-GFP and $\triangle$ Exon27-GFP, and control flies expressing 736 tubulin-Gal4 only. Survivorship was plotted over time. Expression of $\Delta$ Exon27-GFP by the

737 tubulin driver caused a substantial decrease in viability $(p<0.001$, log-rank test). Survival curves

738 represent an average of two trials. (D) Expression of $\triangle$ Exon27-GFP in Drosophila muscles leads

739 to complete paralysis. The pupal case of the fly expressing $\Delta$ Exon27-GFP has been removed for

740 this picture. The folded wings and legs are characteristic of the pupal state in flies expressing

$741 \Delta$ Exon27. (E) Negative geotaxis assay showing reduced motor function in 25-day-old flies

742 expressing $\triangle$ Exon27-GFP driven by a motor neuron specific driver (C380-Gal4) $(\mathrm{n}=50$ each

743 group). Bars indicate mean \pm SD. Statistical analysis was performed using one way-ANOVA.

745 Fig. 6. Proposed mechanisms of neuronal toxicity caused by KIF5A $\Delta$ Exon27 gain of 746 function.

747 In the absence of tethered cargos, KIF5A WT is autoinhibited with the C-terminal tail binding to 748 the N-terminal motor domain. When cargo binds to the C-terminal tail, the motor associates with 749 microtubules and starts a processive run toward the plus-ends. ALS-associated KIF5A $\Delta$ Exon27

750 with the new C-terminal tail relieves from the "autoinhibited" state even without cargo and self751 associates to form multiple motors, leading to a drastically increased run-length on microtubules 752 and accumulating at the plus-ends. $\Delta$ Exon27 also forms complexes with WT KIFA and 753 enhances motor self-association and aggregation. 


\begin{tabular}{|c|c|c|}
\hline Construct & $\begin{array}{c}\text { Velocity }(\text { mean } \pm \\
\text { SD) }(\mu \mathrm{m} / \mathrm{s})\end{array}$ & $\begin{array}{c}\text { Processivity (lifetime } \\
[95 \% \mathrm{CI}])(\mu \mathrm{m})\end{array}$ \\
\hline KIF5A WT-GFP & $0.45 \pm 0.11$ & $0.60[0.51,0.71]$ \\
\hline$\Delta$ Exon27-GFP & $0.66 \pm 0.10$ & $5.47[3.84,7.80]$ \\
\hline$\Delta$ C-GFP & $0.85 \pm 0.07$ & $1.63[1.47,1.81]$ \\
\hline K998-GFP & $0.70 \pm 0.11$ & $2.07[1.72,2.50]$ \\
\hline $\begin{array}{l}\text { KIF5A WT-GFP/AExon27-mCherry } \\
\text { (KIF5A WT-GFP*) }\end{array}$ & $0.62 \pm 0.10$ & $3.95[2.81,5.53]$ \\
\hline $\begin{array}{c}\text { KIF5A WT-GFP/ } \Delta \text { Exon27-mCherry } \\
(\Delta \text { Exon27-mCherry*) }\end{array}$ & $0.62 \pm 0.10$ & $4.49[3.64,5.63]$ \\
\hline
\end{tabular}

Table 1. Measured velocities and run lengths of all KIF5A constructs. The velocity data were fit with Gaussian distribution, and the run length data were fit with an exponential decay function. *Denotes the fluorophore with which KIF5A movement was tracked.

\section{Supplementary Figures}

Fig. S1. KIF5A $\triangle$ Exon27 is prone to form cytoplasmic aggregates. (A-B) Expression of human KIF5A WT and $\triangle$ Exon27 either with C-terminal GFP (A) or N-terminal FLAG tags (B) in N2a cells. (C) Co-localization of G3BP1, p62, and Ubiquitin markers with KIF5A WT expressed in HEK293T cells. (D) Percent of cells with G3BP1, p62 or Ubiquitin markers colocalized with cytoplasmic sexon27 granules. (E) Staining of TDP-43 in HEK293T cells expressing either KIF5A WT or $\triangle$ exon27 showed nuclear TDP-43. (F) Immunoprecipitation of KIF5A using antibodies against the N-terminal FLAG tag also pulled down endogenous p62

Fig. S2. KIF5A $\Delta$ Exon 27 accumulates at the plus-ends of microtubules. (A) Primary neurons expressing KIF5A $\Delta$ exon27 stained with pre-synaptic marker synapsin I. (B) Expression of KIF5A WT-mApple and $\Delta$ exon27-mApple in LLC-PK1 cells stably expressing tubulin-GFP. KIF5A $\Delta$ exon27 showed aggregation at proximal tubule regions as shown by white arrowhead. 

K998 and $\Delta$ C. (A) Schematic illustrations of KIF5A truncated mutants, K998 and $\Delta$ C. (B) Both KIF5A K998 and $\Delta \mathrm{C}$ truncated proteins diffuse in the cytoplasm when expressed in HEK293T and N2a cells. (C) Motor landing rate of KIF5A $\triangle$ Exon27 along the microtube was accessed. $\Delta$ Exon27 does not have a preference over the landing location on microtubules. (D) Motor velocities (middle) and run lengths (right) were determined based on kymographs (example on the left) for KIF5A WT and $\triangle$ Exon27 complexes. HEK293T cells were transfected with both KIF5A WT-GFP and $\triangle$ Exon27-mApple. The movements of WT and $\Delta$ Exon27 were assessed by tracking either GFP or mApple, respectively. The right kymograph in $D$ is the overlay of the GFP and mApple of moving WT-GFP/AExon27-mApple complexes. The x-axis scale for the processivity graphs was limited to $20 \mu \mathrm{m}$ to permit a direct comparison of the run lengths of the different constructs. Diagonal lines in the kymograph represent KIF5A molecules moving over time. The velocity data were fit with Gaussian distribution and the processivity data were fit with an exponential decay function. The measured values for the velocities and run lengths are listed in Table 1.

Fig. S4. Enhanced motor self-association in KIF5A $\Delta$ Exon27. (A) Histograms of the measured fluorescence intensities of the various moving kinesin constructs. The fluorescence intensity in every frame of each moving spot was measured, accumulated, and plotted in a

794 histogram. Moving KIF5A $\triangle$ Exon27 molecules exhibit a much larger fraction of high 795 fluorescence intensities. The majority of the moving KIF5A $\Delta$ Exon27 spots consist of multiple motors. (B) Two example traces of K490-EmGFP showing photobleaching events. K490-emGFP was used as the standard dimer molecule to determine the fluorescent intensity of EmGFP. (C)

798 Histogram of measured intensities of moving K490-EmGFP molecules that showed a single photobleaching step. The intensity distribution was fit with two Gaussian functions, resulting in

800 mean values of $145.5 \pm 3.6$ (mean \pm SD) and $163.0 \pm 7.3$. With an average background 801 fluorescence signal of $127.6 \pm 0.7$, one obtains an average fluorescent intensity is 17.7 for 802 EmGFP (in a.u.). (D-E) Determination of the percentage of the number of motors for each motor 803 construct. KIF5A K490 and KIF5A $\triangle$ Exon27 are depicted here as examples (D). The same 804 method was applied to the other constructs. Each moving spot was assigned to a single intensity 805 value that corresponded to the average intensity measured over the first 20 frames of the moving 
806 spot so the intensity would be less likely to be averaged down due to photobleaching. The

807 measured values were then accumulated and plotted in a histogram. Based on the mean intensity

808 of EmGFP, the measured intensity values were sorted into intervals of 35.4 (depicted by the

809 turquoise dashed lines), with the first interval centered at 163 (163 \pm 17.7$)$. The percentage of

810 each bin was calculated and then assigned to the calculated number of dimers (E). The

811 percentages of a single dimer for all KIF5A constructs are: K490, 100\%; $\Delta \mathrm{C}, 88 \%$; K998, 54\%;

812 WT, 54\%; $\Delta$ Exon27, 25\%.

813

814 Fig. S5. Comparison of amino acid sequences in the C-terminal tail domains of ALS- and 815 NEIMY- associated KIF5A. The sequences in red are the new 39 amino acids of the C-terminal 816 tail in ALS-associated KIF5A caused by mis-splicing of exon 27 (AExon27). The amino acids in 817 blue and green are those different from WT caused by a single nucleotide deletion in three 818 NEIMY cases.

Fig. S6. Muscle-specific expression of $\Delta$ Exon27 in Drosophila leads to early lethality. (A)

821 Western blot showing expression of human KIF5A proteins in Drosophila ubiquitously 822 expressing WT or $\Delta$ Exon27 driven by tubulin-Gal4. Genotypes were: KIF5A WT (UAS-KIF5A 823 WT-GFP/ tubulin-Gal4); $\Delta$ Exon27 (UAS- $\triangle$ Exon27-GFP/ tubulin-Gal4). Samples from 4 (WT) 824 and 5 ( $\Delta$ Exon27) different induvial lines were assessed with similar results. (B) Western blot 825 showing expression of human KIF5A protein in Drosophila expressing WT or $\Delta$ Exon27 in 826 muscle tissues driven by MHC-Gal4. Genotypes were: Control (MHC-Gal4/+); KIF5A WT 827 (UAS-KIF5A WT-GFP/MHC-Gal4); $\Delta$ Exon27 (UAS- $\Delta$ Exon27-GFP/MHC-Gal4) (C) Lifespan 828 of male flies expressing KIF5A WT or $\triangle$ Exon27 in muscles driven by MHC-Gal4 at $25{ }^{\circ} \mathrm{C}$. 


\section{Figures for}

\section{ALS-linked KIF5A $\Delta$ exon27 mutant causes neuronal toxicity through gain of function}

Devesh C. Pant ${ }^{1 \dagger}$, Janani Parameswaran ${ }^{1 \dagger}$, Lu Rao ${ }^{2}$, Liang Shi ${ }^{1}$, Ganesh Chilukuri ${ }^{1}$, Zachary T. McEachin $^{1,3}$, Jonathan Glass ${ }^{4}$, Gary J. Bassell ${ }^{1}$, Arne Gennerich ${ }^{2}$, Jie Jiang ${ }^{1 *}$

*Corresponding author. Email: jie.jiang@emory.edu

\section{This file includes:}

Figs. 1 to 6 
A

\begin{tabular}{|c|c|}
\hline $\begin{array}{c}\text { Motor } \\
\text { (1-327aa) }\end{array}$ & $\begin{array}{c}\text { Tail } \\
\text { (331-906aa) }\end{array}$ \\
\hline
\end{tabular}

$\underline{\Delta E x o n} 27$

\begin{tabular}{ccc}
\hline $\begin{array}{c}\text { Motor } \\
(1-327 \text { aa })\end{array}$ & $\begin{array}{c}\text { Stalk } \\
(331-906 a a)\end{array}$ & $\begin{array}{c}\text { Tail } \\
(907-1037 a a)\end{array}$ \\
\hline
\end{tabular}

B

HEK293T cells (24 hours)
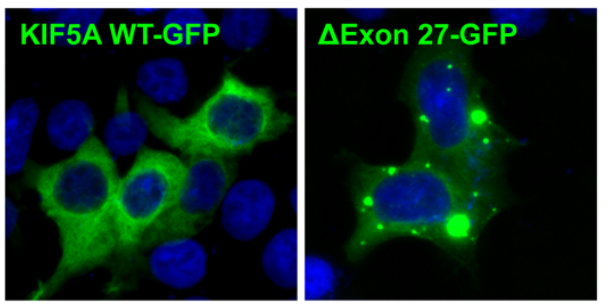

HEK293T cells (48 hours)
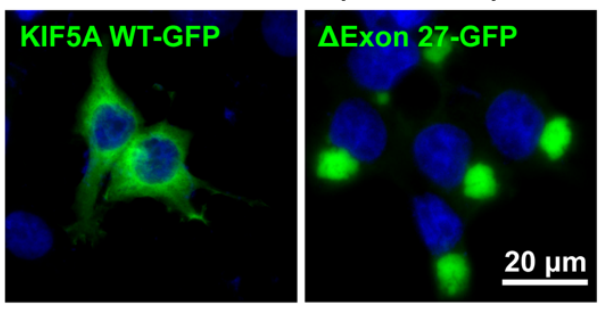

D

RIPA soluble
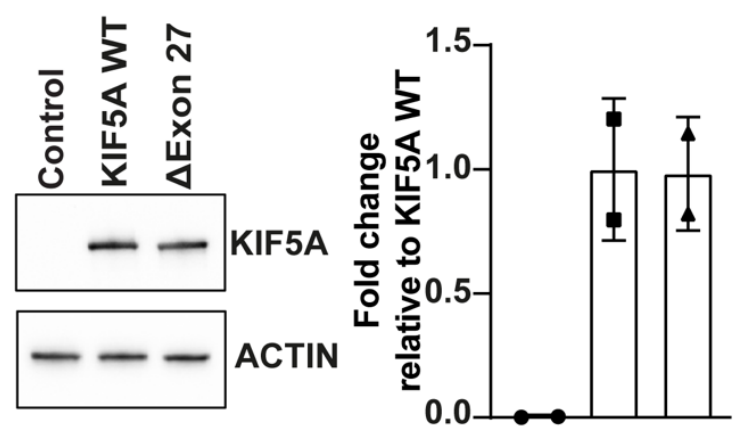

KIF5A WT

\begin{tabular}{l|l|l|l|l|}
\hline Normal spliced mRNA 26 & 27 & 28 & 29 \\
\hline
\end{tabular}

Translation stop

Normal protein:

MDNGNATDINDNRSDLPCGYEAEDQAKLFPLHQETAAS*

\section{$\Delta$ Exon27}

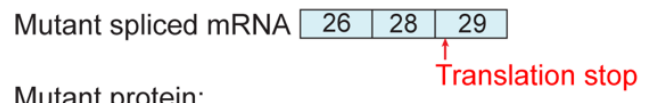

MDNGVTCRVAMRLRTRPSFSLSTKRQQPANLPHPRLHTCTFSF*

C
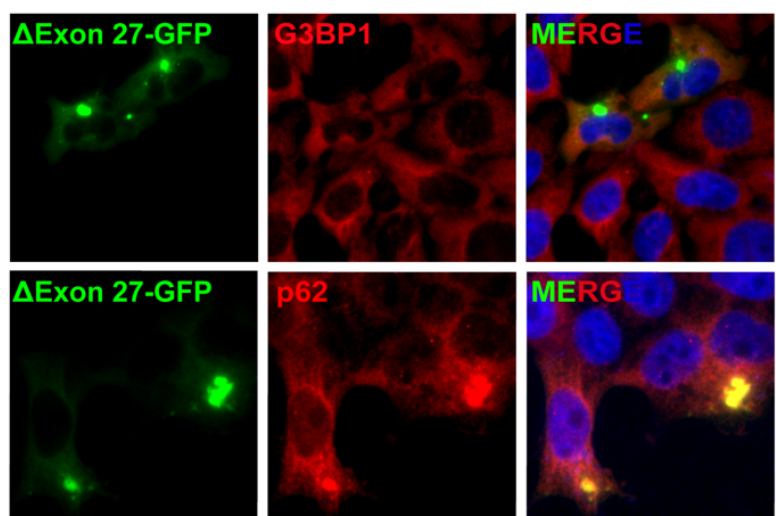

MERG
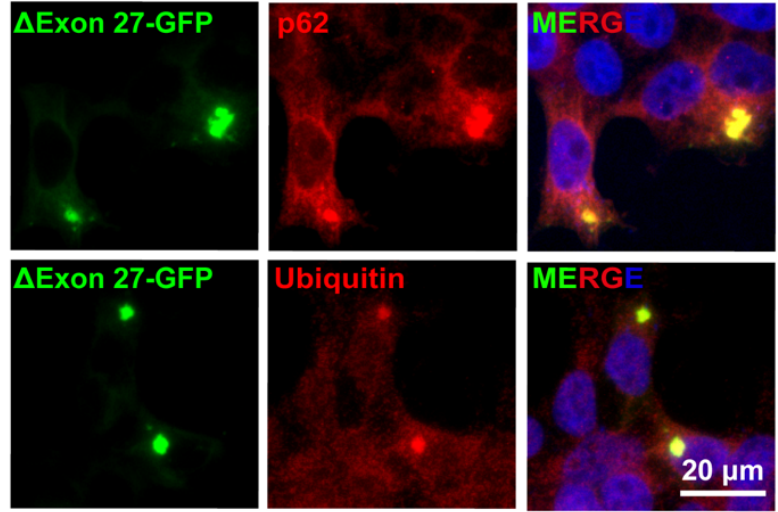

E

Urea soluble
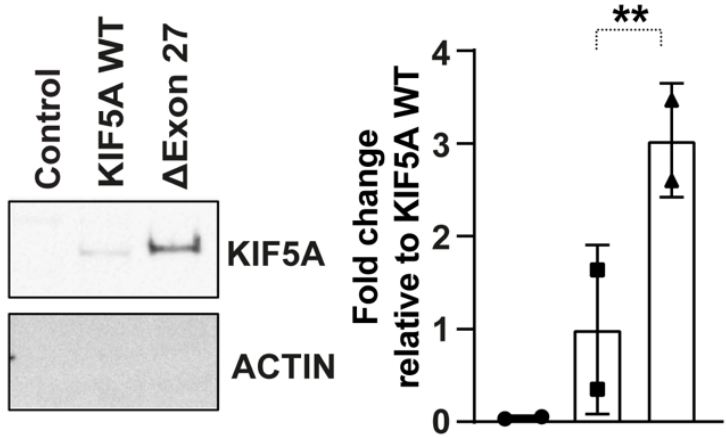

\section{FIGURE 1}


A
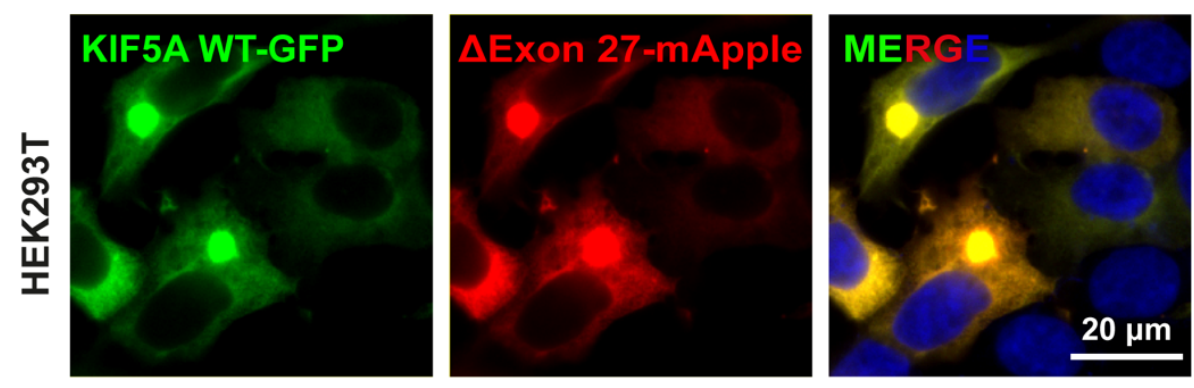

B
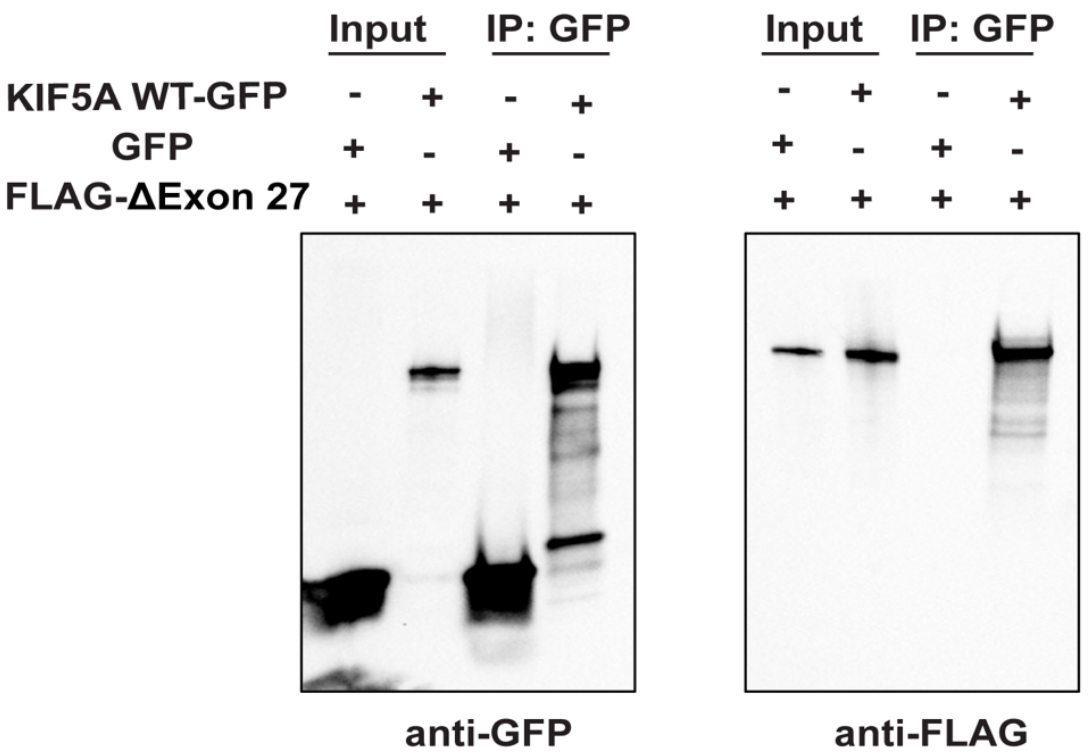

C
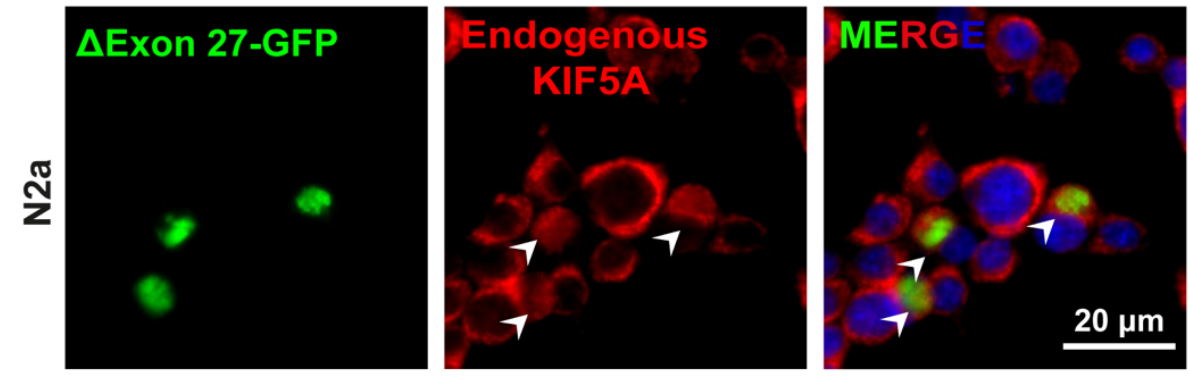

FIGURE 2 
bioRxiv preprint doi: https://doi.org/10.1101/2022.03.05.483071; this version posted March 7, 2022. The copyright holder for this preprint (which was not certified by peer review) is the author/funder, who has granted bioRxiv a license to display the preprint in perpetuity. It is made available under aCC-BY-NC-ND 4.0 International license.
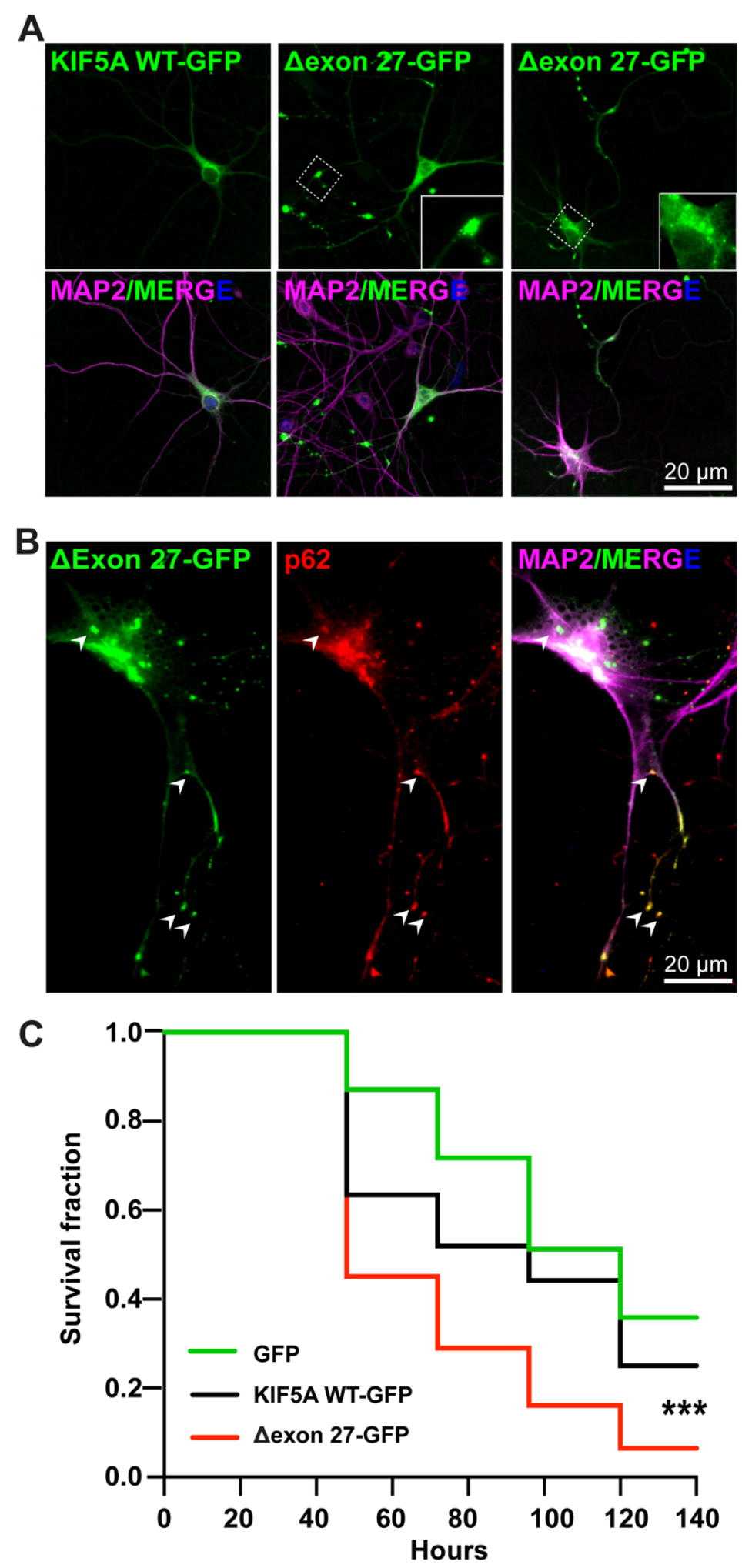

FIGURE 3 
A

B

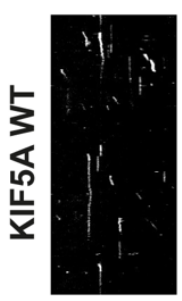

C
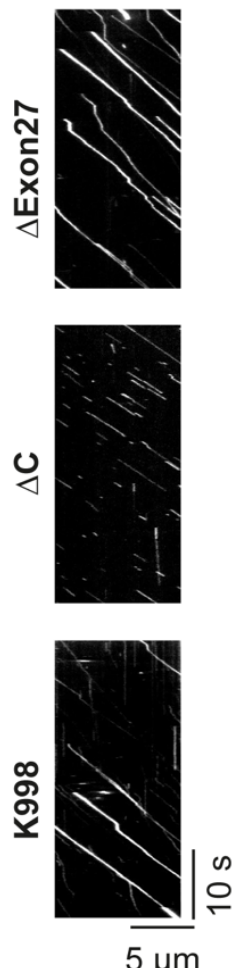
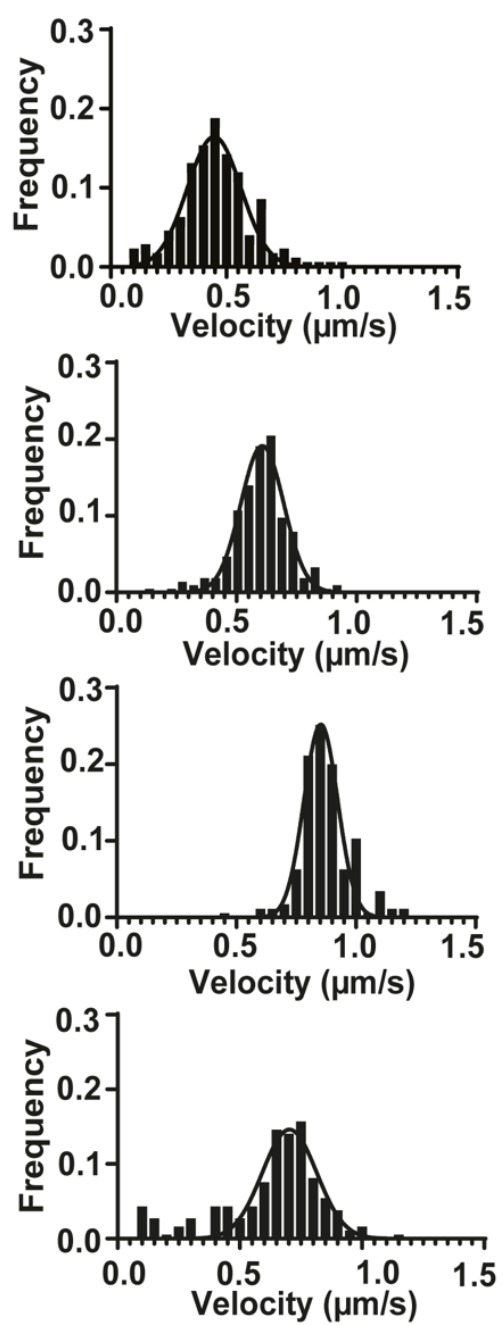
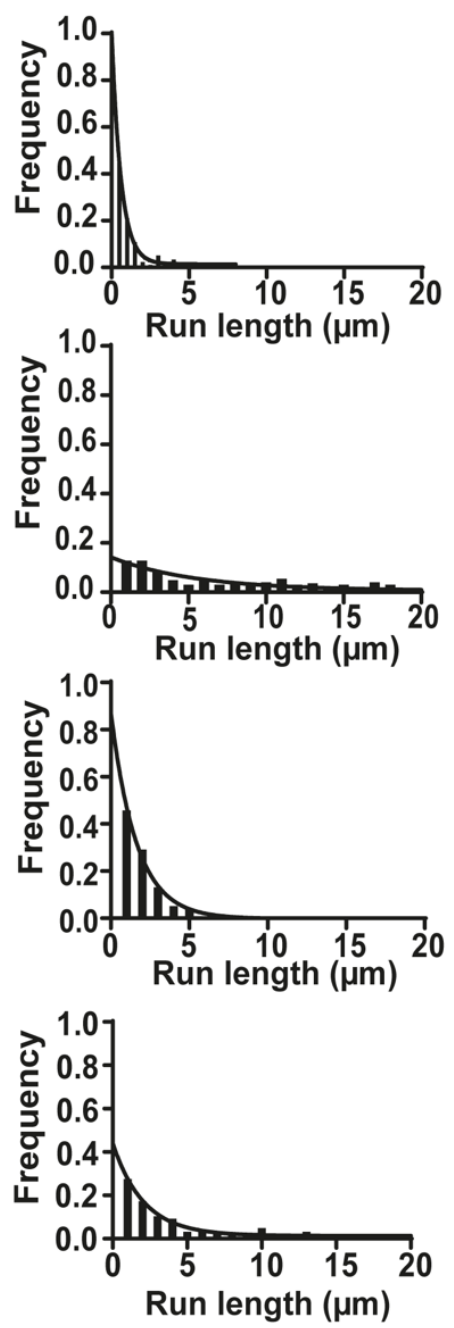
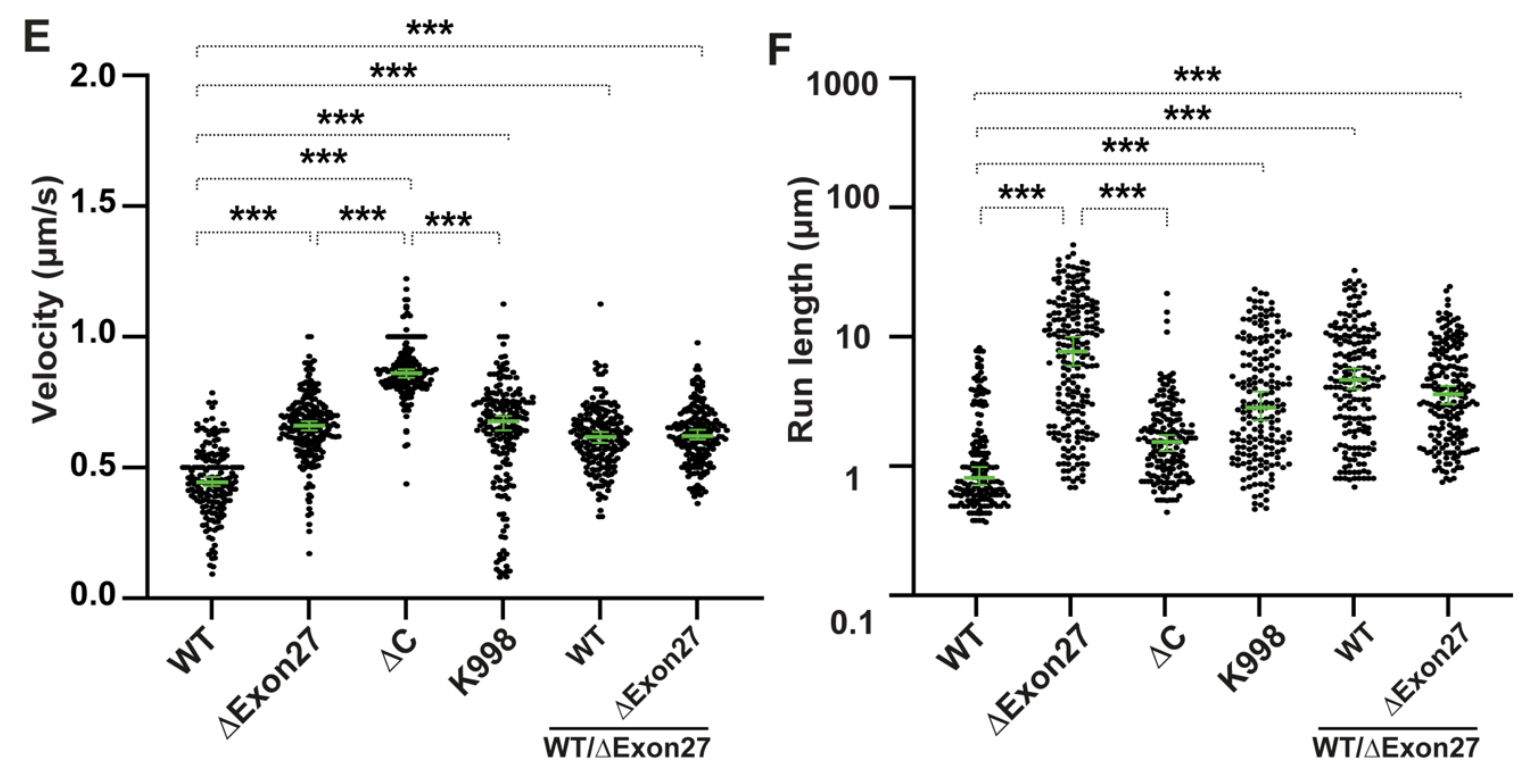

FIGURE 4 
A

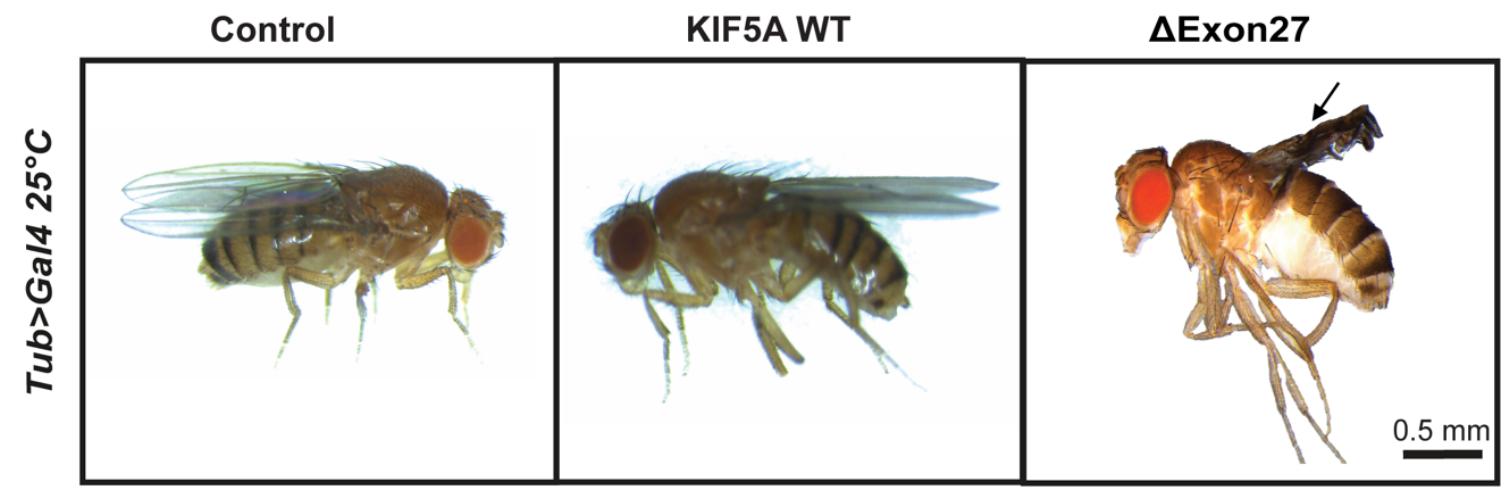

B

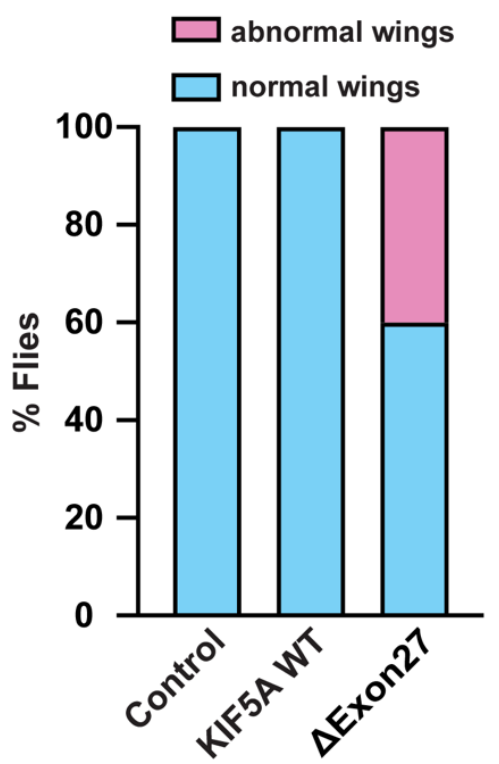

C

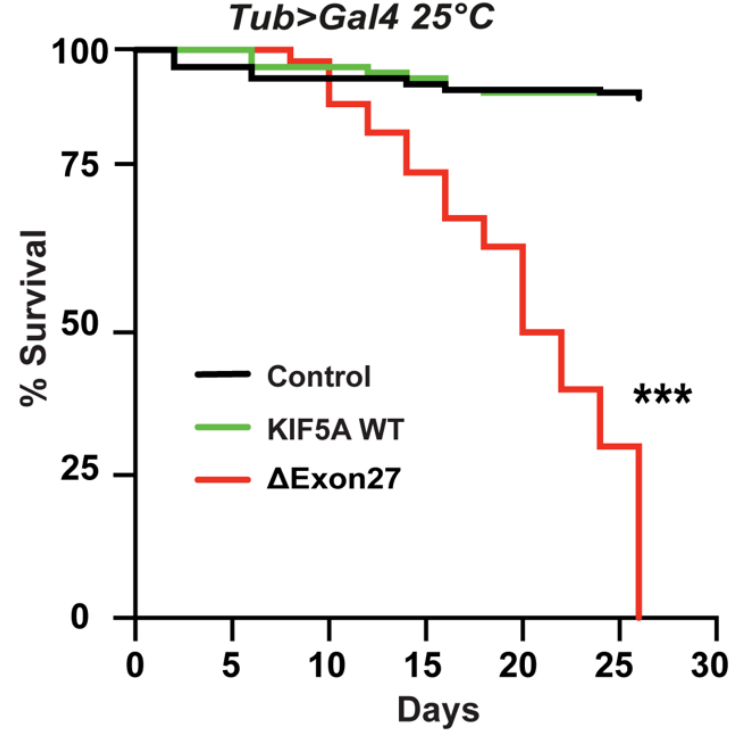

D

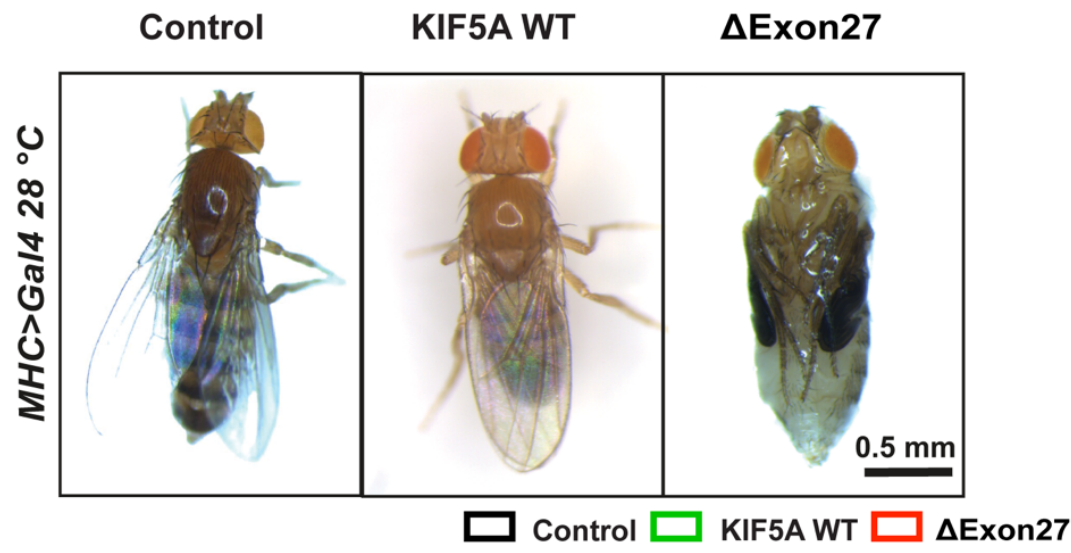

$\mathbf{E}$

C $380>$ Gal4 $28^{\circ} \mathrm{C}$

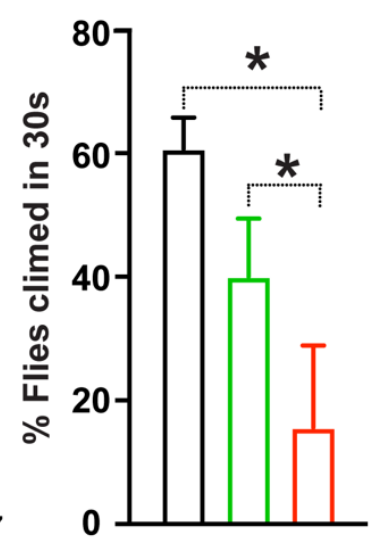

FIGURE 5 


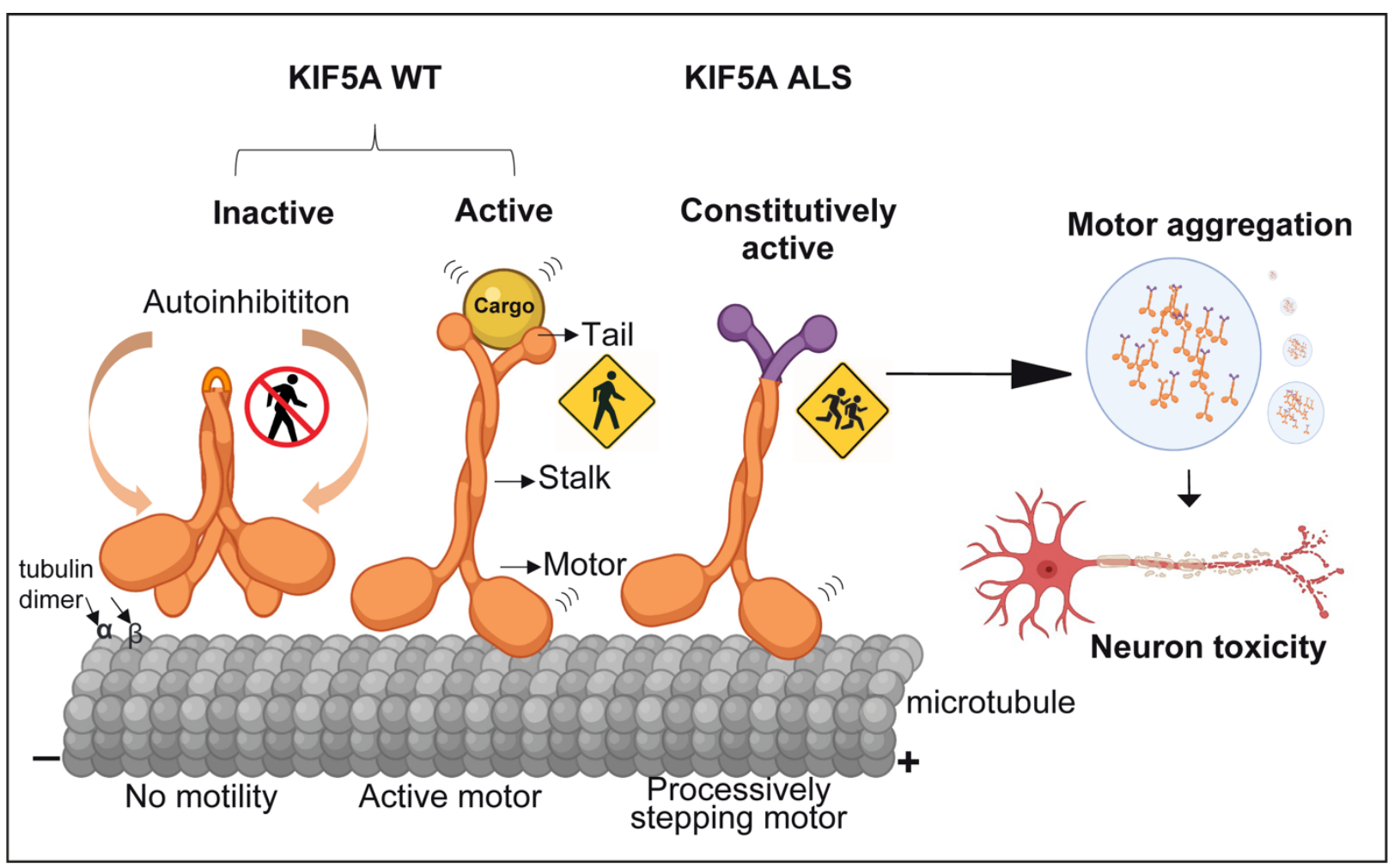

FIGURE 6 


\section{Supplementary Materials for}

\section{ALS-linked KIF5A $\Delta$ exon27 mutant causes neuronal toxicity through gain of function}

Devesh C. Pant ${ }^{1 \dagger}$, Janani Parameswaran ${ }^{1 \dagger}$, Lu Rao ${ }^{2}$, Liang $\mathrm{Shi}^{1}$, Ganesh Chilukuri ${ }^{1}$, Zachary T. McEachin $^{1,3}$, Jonathan Glass ${ }^{4}$, Gary J. Bassell ${ }^{1}$, Arne Gennerich ${ }^{2}$, Jie Jiang ${ }^{{ }^{*}}$

*Corresponding author. Email: jie.jiang@emory.edu

This file includes:

Figs. S1 to S6 
Fig. S1

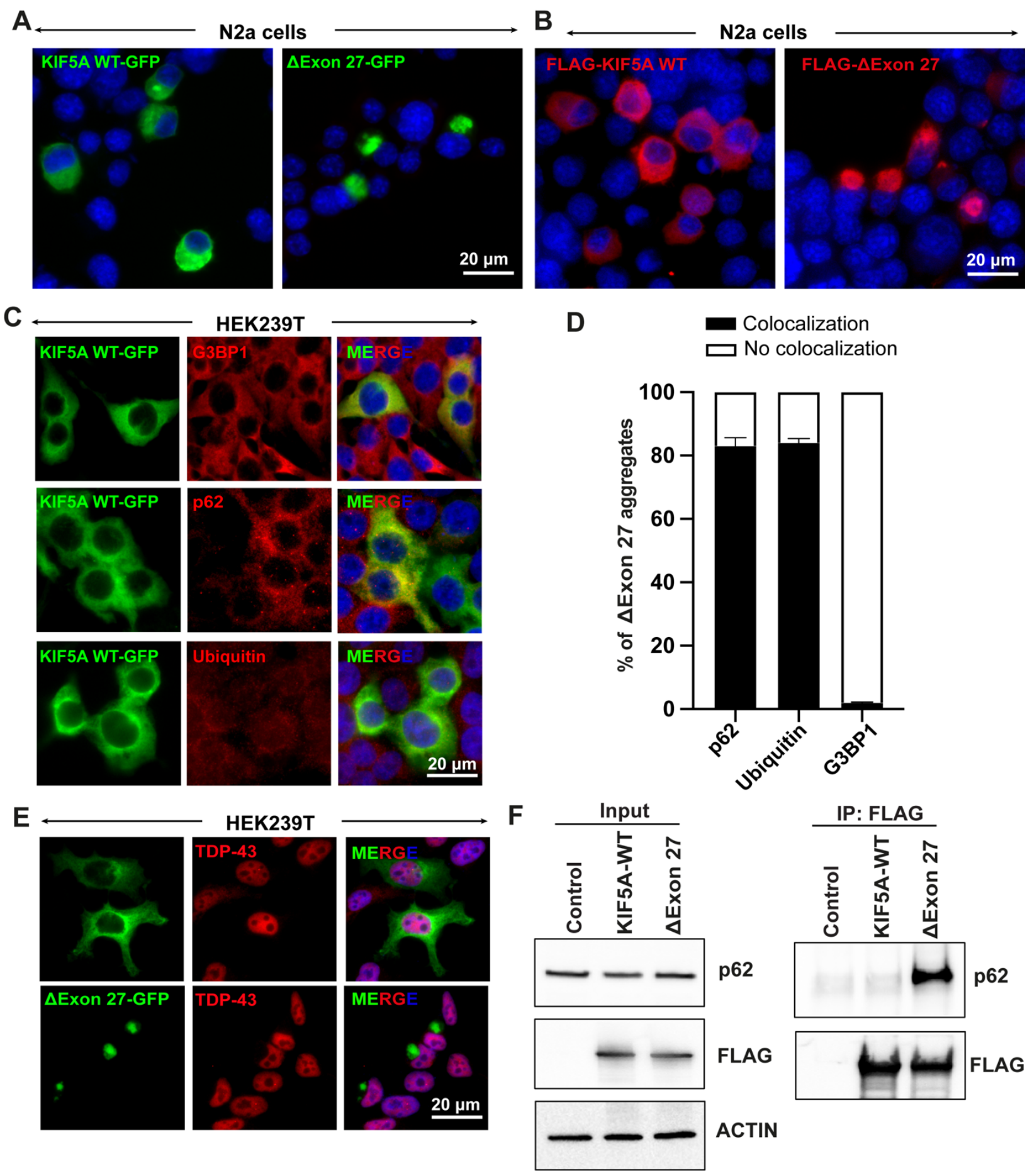


bioRxiv preprint doi: https://doi.org/10.1101/2022.03.05.483071: this version posted March 7, 2022. The copyright holder for this preprin (which was not certified by peer review) is the author/funder, who has granted bioRxiv a license to display the preprint in perpetuity. It is made available under aCC-BY-NC-ND 4.0 International license.

Fig. S2

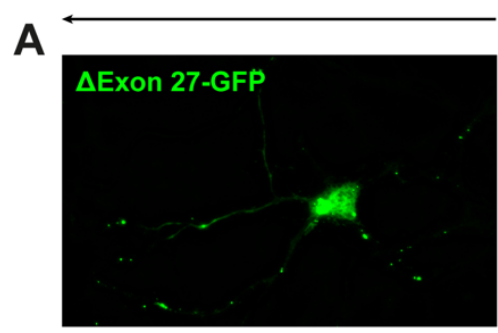

\section{Primary cortical neurons}
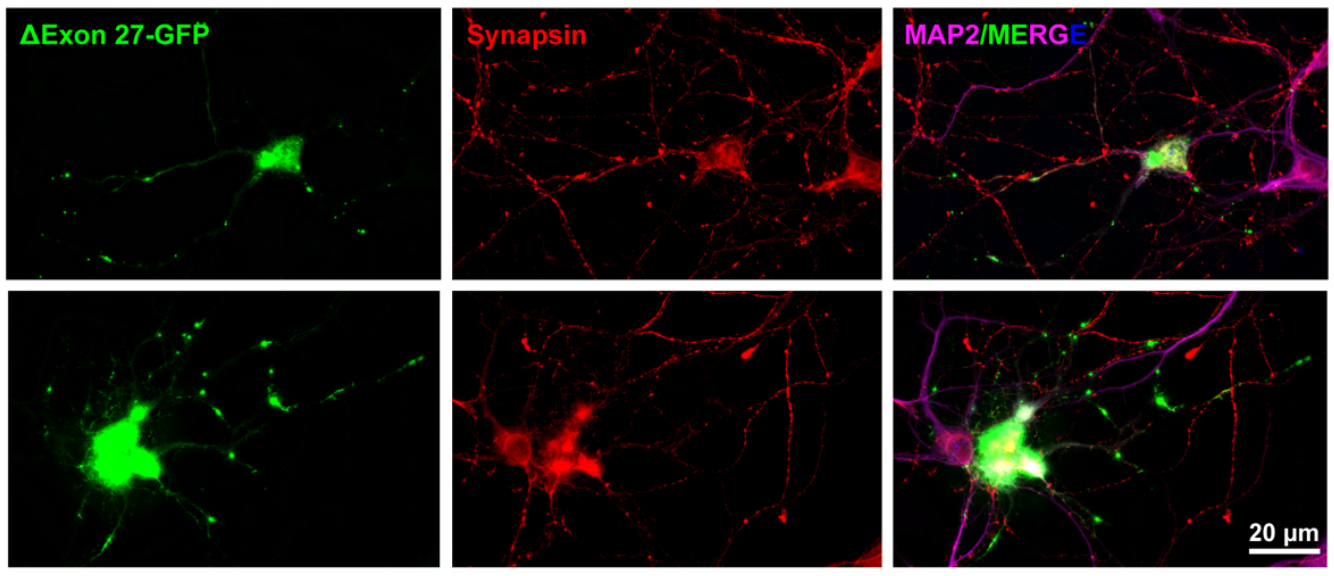

\section{B}
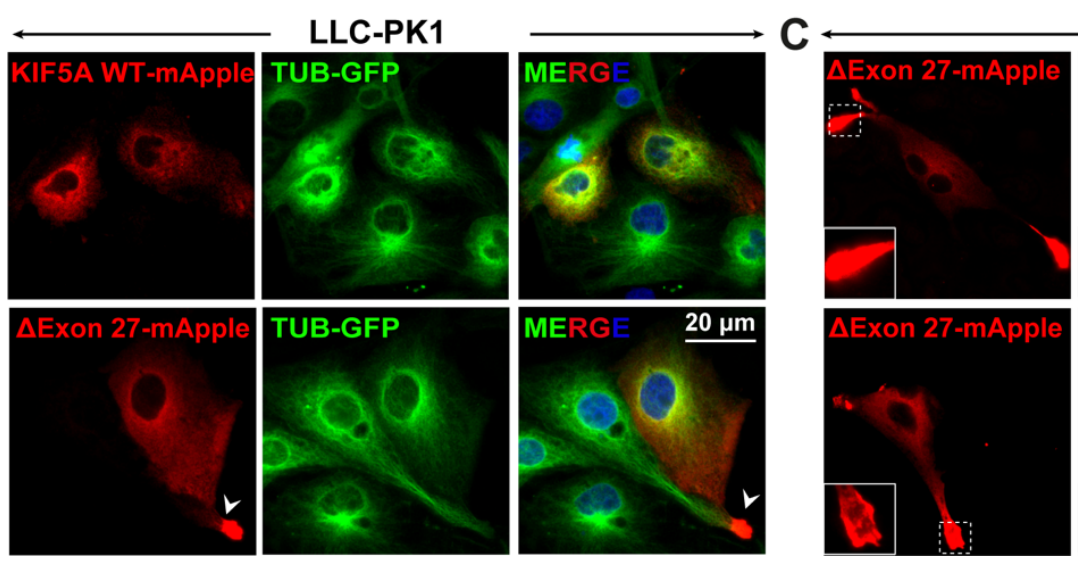

LLC-PK1
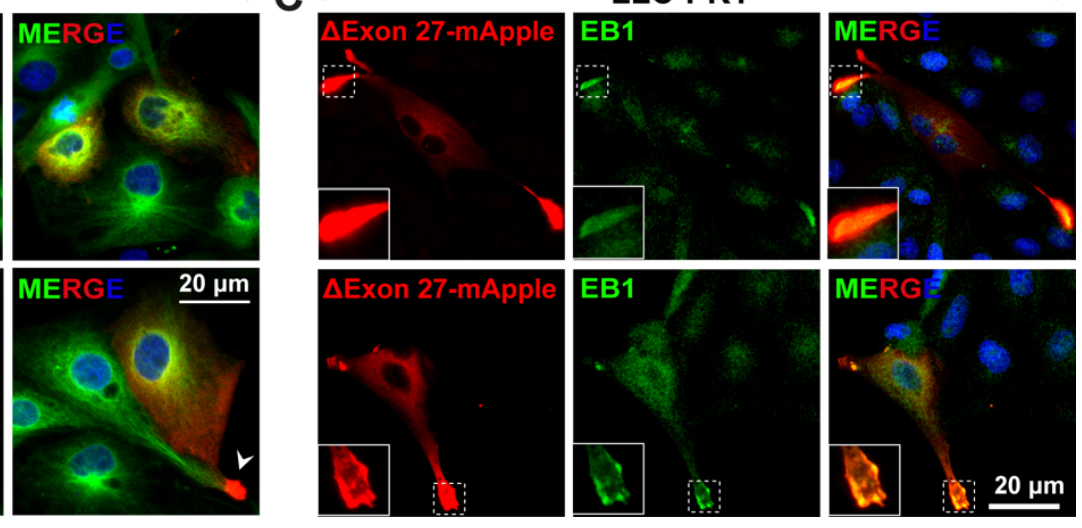
Fig. S3

A
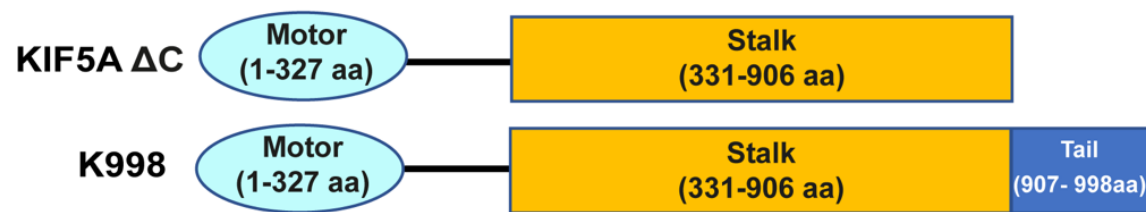

B
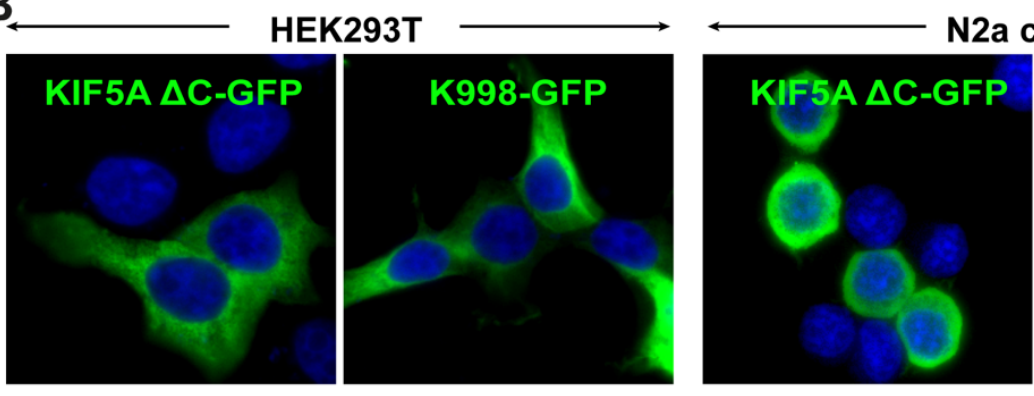

N2a cells

C
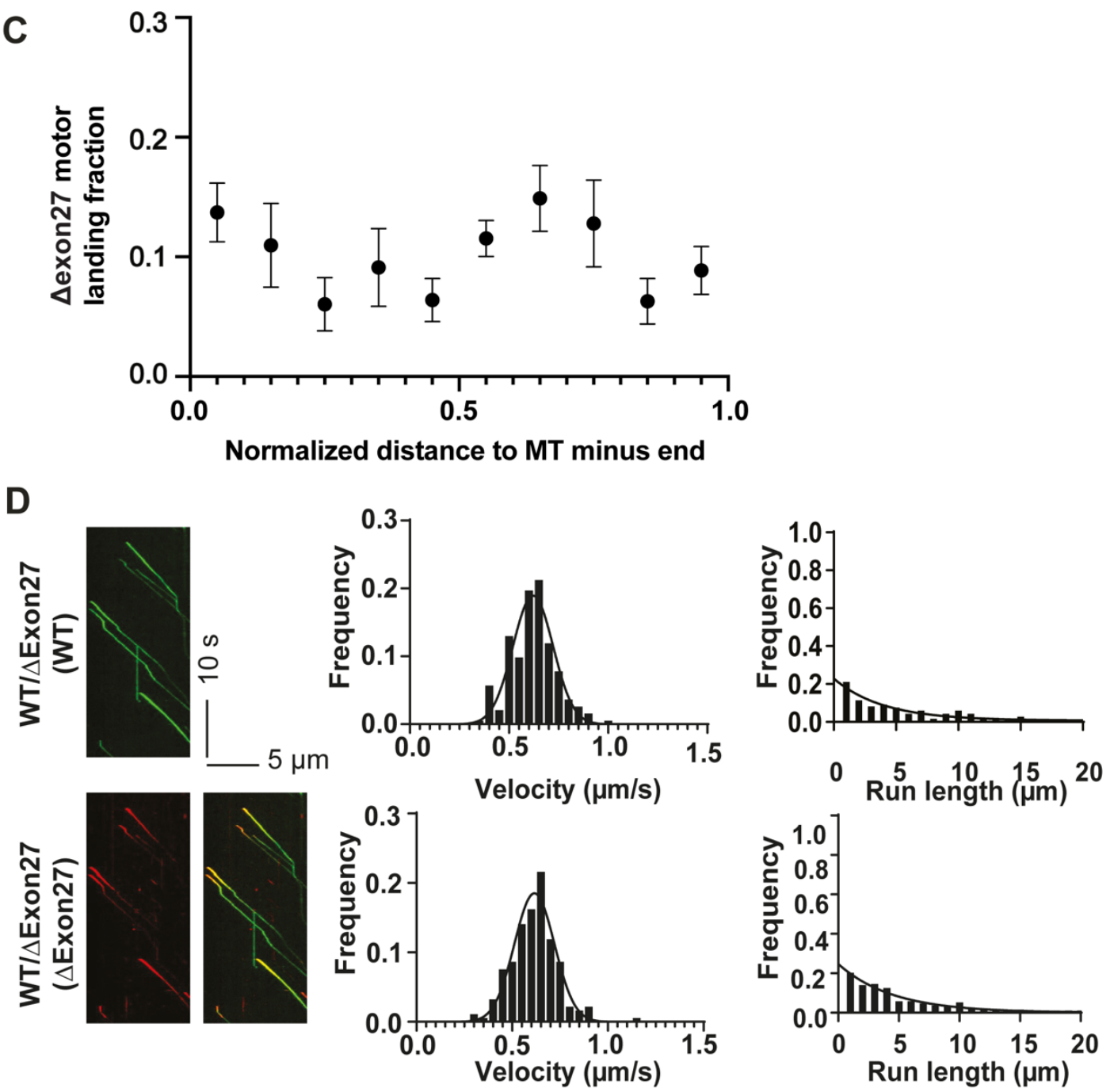
Fig. S4
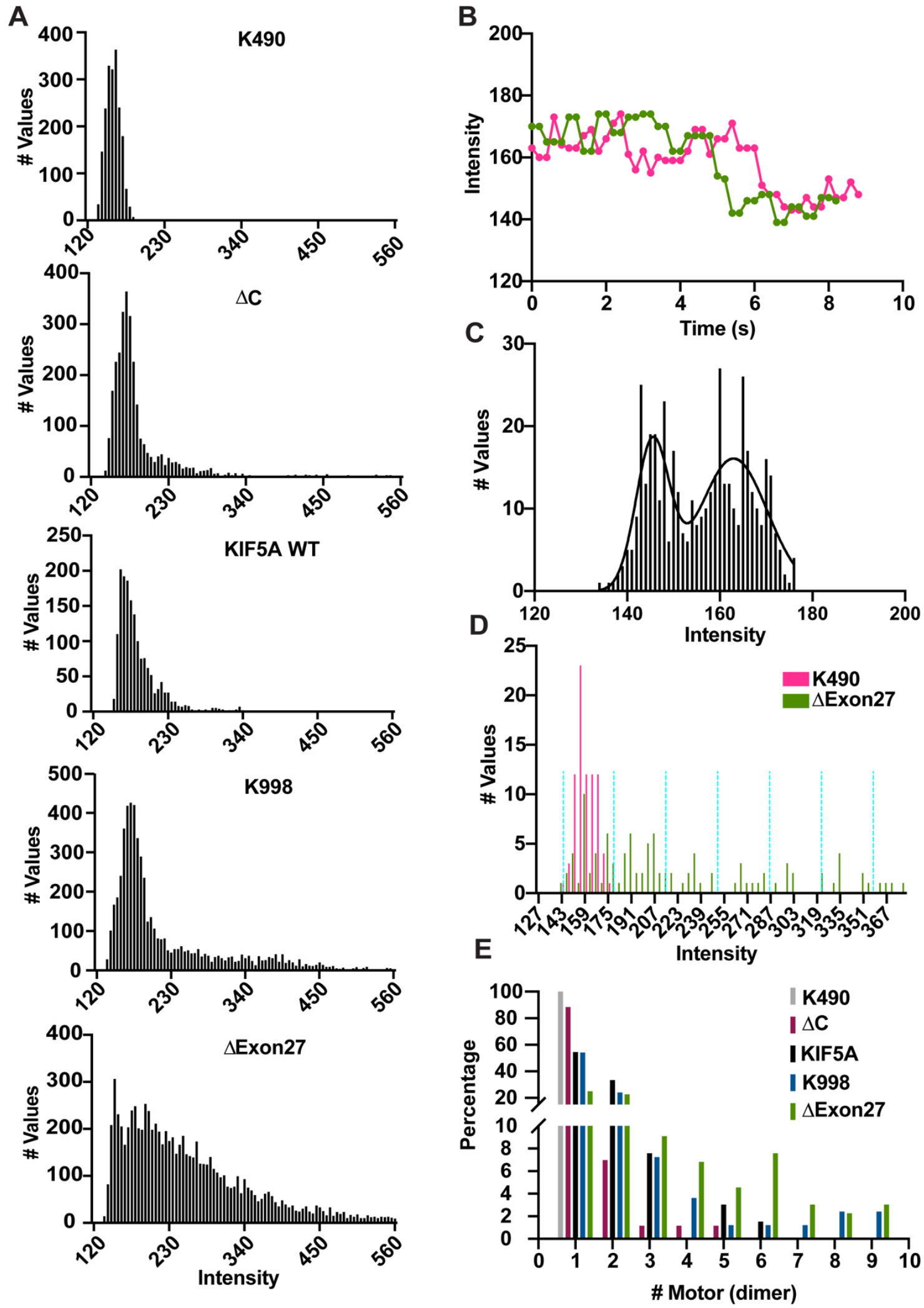
bioRxiv preprint doi: https://doi.org/10.1101/2022.03.05.483071; this version posted March 7, 2022. The copyright holder for this preprint (which was not certified by peer review) is the author/funder, who has granted bioRxiv a license to display the preprint in perpetuity. It is made available under aCC-BY-NC-ND 4.0 International license.

\section{Fig. S5}

907

KIF5A Tail Domain

\begin{tabular}{|c|c|c|}
\hline & 07 \\
\hline & Wild-type & KSSGKRGHSAQIAKPVRPGHYPASSPTNPYGTRSPECISYTNSLFQNYQNLYLQATPSSTSDMY \\
\hline \multirow{4}{*}{$\underline{\mathrm{ALS}}$} & Splicing variants ( $\Delta$ exon27) & KSSGKRGHSAQIAKPVRPGHYPASSPTNPYGTRSPECISYTNSLFQNYQNLYLQATPSSTSDMY \\
\hline & c.2996delA (p.Asn999fs) & KSSGKRGHSAQIAKPVRPGHYPASSPTNPYGTRSPECISYTNSLFQNYQNLYLQATPSSTSDMY \\
\hline & c.2989delA (p.Asn997fs) & KSSGKRGHSAQIAKPVRPGHYPASSPTNPYGTRSPECISYTNSLFQNYQNLYLQATPSSTSDMY \\
\hline & c.2987delA (p.Asp996fs) & KSSGKRGHSAQIAKPVRPGHYPASSPTNPYGTRSPECISYTNSLFQNYQNLYLQATPSSTSDMY \\
\hline \multirow{3}{*}{ NEIMY } & c.2934delG (p.Ser978fs) & KSSGKRGHSAQIAKPVRPGHYPASSPTNPYGTRSPECISYTNSLFQNYQNLYLQATPSSTSDMY \\
\hline & c.2921delC (p.Ser974fs) & KSSGKRGHSAQIAKPVRPGHYPASSPTNPYGTRSPECISYTNSLFQNYQNLYLQATPSSTSDMY \\
\hline & c.2854delC (p.GIn952fs) & KSSGKRGHSAQIAKPVRPGHYPASSPTNPYGTRSPECISYTNSLFR TTR ISTCRPHPAPPQICT \\
\hline
\end{tabular}

971

Wild-type FANSCTSSGATSSGGPLASYQKANMDNGNATDINDNRSDLPCGYEAEDQAKLFPLHQETAAS*
$\underline{\text { ALS }}\left\{\begin{aligned} \Delta \text { exon27 } & \text { FANSCTSSGATSSGGPLASYQKANMDNGVTCRVAMRLRTRPSFSLSTKRQQPANLPHPRLHTCTFSF* } \\ \text { c.2996delA } & \text { FANSCTSSGATSSGGPLASYQKANMDNGMPQISMTIGVTCRVAMRLRTRPSFSLSTKRQQPANLPHPRLHTCTFSF* } \\ \text { c.2989delA } & \text { FANSCTSSGATSSGGPLASYQKANMDMEMPQISMTIGVTCRVAMRLRTRPSFSLSTKRQQPANLPHPRLHTCTFSF* } \\ \text { c.2987delA } & \text { FANSCTSSGATSSGGPLASYQKANMTMEMPQISMTIGVTCRVAMRLRTRPSFSLSTKRQQPANLPHPRLHTCTFSF* }\end{aligned}\right.$
$\underline{\text { NEIMY }} \begin{cases}\text { c.2934delG } & \text { FANSCTSSGATLLAAPWLPTRRPTWTMEMPQISMTIGVTCRVAMRLRTRPSFSLSTKRQQPANLPHPRLHTCTFSF* } \\ \text { c.2921deIC } & \text { FANSVPAVEPHLLAAPWLPTRRPTWTMEMPQISMTIGVTCRVAMRLRTRPSFSLSTKRQQPANLPHPRLHTCTFSF* } \\ \text { c.2854delC } & \text { LQTPVPAVEPHLLAAPWLPTRRPTWTMEMPQISMTIGVTCRVAMRLRTRPSFSLSTKRQQPANLPHPRLHTCTFSF* }\end{cases}$ 


\section{Fig. S6}

A

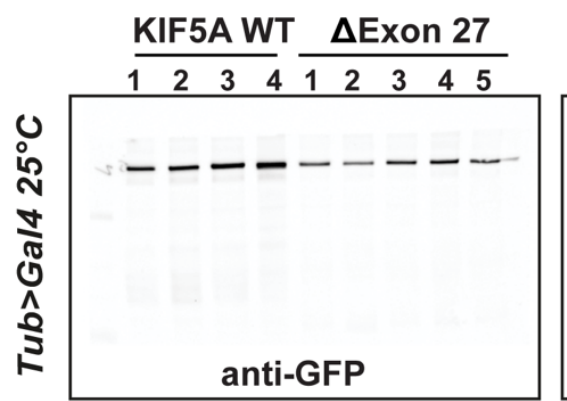

KIF5A WT $\Delta$ Exon 27

KIF5A WT $\quad \Delta$ Exon 27

$\begin{array}{lllllllll}1 & 2 & 3 & 4 & 1 & 2 & 3 & 4 & 5\end{array}$

$\begin{array}{lllllllll}12 & 3 & 4 & 2 & 345\end{array}$
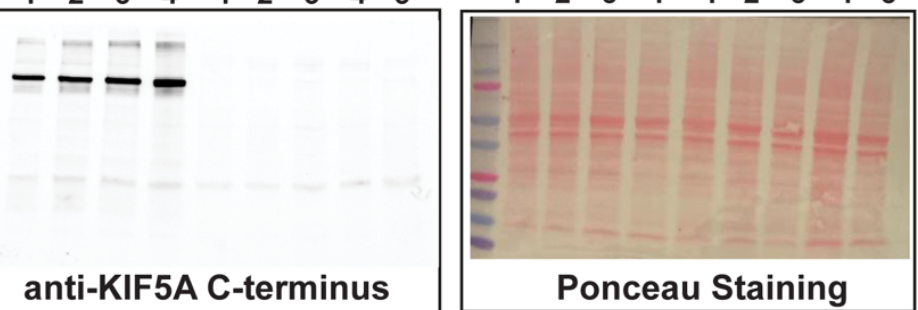

B $M H C>G a l 425^{\circ} \mathrm{C}$

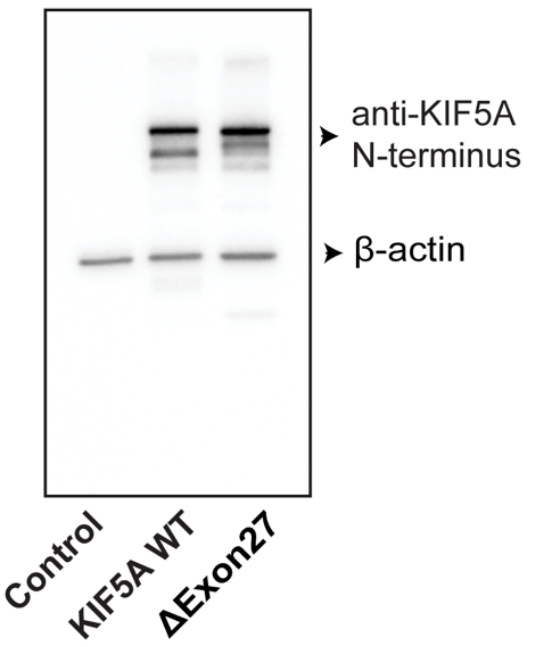

C

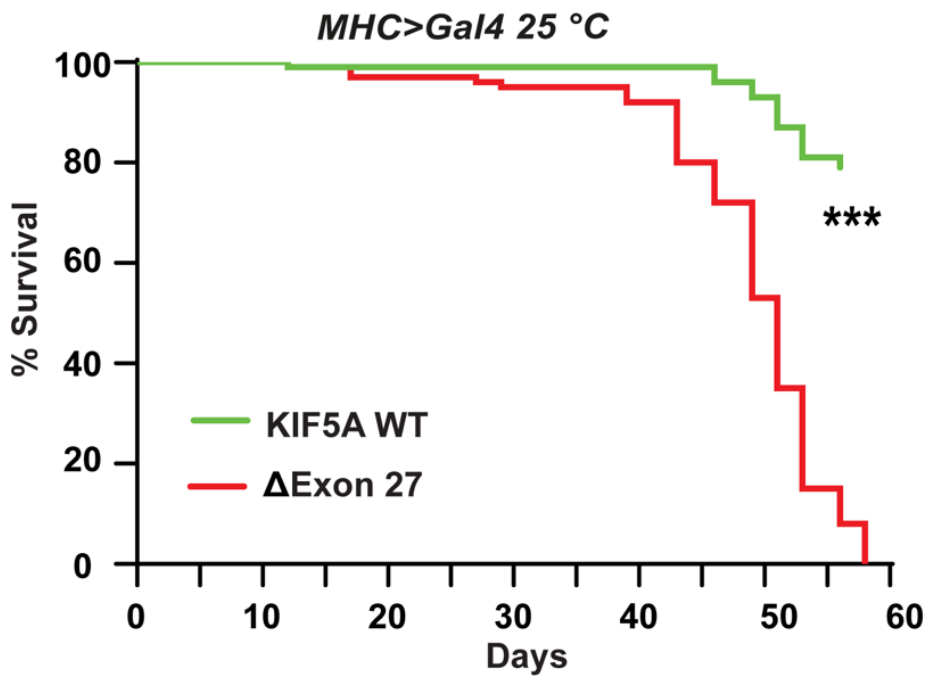

